NBER WORKING PAPER SERIES

\title{
SUPPLY AND DEMAND IN DISAGGREGATED KEYNESIAN ECONOMIES WITH AN APPLICATION TO THE COVID-19 CRISIS
}

\author{
David Baqaee \\ Emmanuel Farhi \\ Working Paper 27152 \\ http://www.nber.org/papers/w27152 \\ NATIONAL BUREAU OF ECONOMIC RESEARCH \\ 1050 Massachusetts Avenue \\ Cambridge, MA 02138 \\ May 2020, Revised February 2021
}

Emmanuel Farhi tragically passed away in July, 2020. Emmanuel was a one-in-a-lifetime friend and collaborator. Finishing this paper without him has been very difficult. We thank Veronica De Falco, Stephanie Kestelman, and Sihwan Yang for excellent research assistance. We thank Andy Atkeson, Natalie Bau, Jennifer La'O, and the editor and referees for their comments. The authors received support from NSF grant No. 1947611. The views expressed herein are those of the authors and do not necessarily reflect the views of the National Bureau of Economic Research.

NBER working papers are circulated for discussion and comment purposes. They have not been peer-reviewed or been subject to the review by the NBER Board of Directors that accompanies official NBER publications.

(C) 2020 by David Baqaee and Emmanuel Farhi. All rights reserved. Short sections of text, not to exceed two paragraphs, may be quoted without explicit permission provided that full credit, including (๑) notice, is given to the source. 
Supply and Demand in Disaggregated Keynesian Economies with an Application to the Covid-19 Crisis

David Baqaee and Emmanuel Farhi

NBER Working Paper No. 27152

May 2020, Revised February 2021

JEL No. E0,E12,E2,E23,E24,E3,E52,E6,E62

\begin{abstract}
We study supply and demand shocks in a disaggregated model with multiple sectors, multiple factors, input-output linkages, downward nominal wage rigidities, credit-constraints, and a zero lower bound. We use the model to understand how the Covid-19 crisis, an omnibus supply and demand shock, affects output, unemployment, and inflation, and leads to the coexistence of tight and slack labor markets. We show that negative sectoral supply shocks are stagflationary, whereas negative demand shocks are deflationary, even though both can cause Keynesian unemployment. Furthermore, complementarities in production amplify Keynesian spillovers from supply shocks but mitigate them for demand shocks. This means that complementarities reduce the effectiveness of aggregate demand stimulus. In a stylized quantitative model of the US, we find supply and demand shocks each explain about half the reduction in real GDP from February to May, 2020. Although there was as much as $7 \%$ Keynesian unemployment, this was concentrated in certain markets. Hence, aggregate demand stimulus is one quarter as effective as in a typical recession where all labor markets are slack.
\end{abstract}

\author{
David Baqaee \\ Department of Economics \\ University of California at Los Angeles \\ Bunche Hall \\ Los Angeles, CA 90095 \\ and CEPR \\ and also NBER \\ baqaee@econ.ucla.edu \\ Emmanuel Farhi \\ and CEPR
}




\section{Introduction}

Covid-19 is an unusual macroeconomic shock. It cannot easily be categorized as an aggregate supply or demand shock. Rather, it is a messy combination of disaggregated sectoral supply and demand shocks. These shocks propagate through supply chains to create different cyclical conditions in different parts of the economy. Some sectors are tight, constrained by supply constraints, and struggling to keep up with demand. Other sectors are slack and shedding workers to reduce excess capacity because of lack of demand.

Separating demand shortfalls from supply constraints is important because supplyand demand-constrained sectors respond very differently to policies. For example, policies that boost demand, like lowering interest rates or increasing government spending, exacerbate problems of inadequate supply, leading to shortages and inflation. Similarly, policies that boost supply, like relaxing lock-downs or providing liability exemptions, are ineffective at restoring activity when applied to demand-constrained sectors.

We model the outbreak of the pandemic as a combination of supply and demand shocks. We define demand shocks to be changes in households' indifference curves over goods, and we define supply shocks to be changes in the economy's production possibilities. Clearly, the Covid-19 crisis contained elements of both shocks.

On the one hand, even fixing budget constraints, households rebalanced their current expenditures across sectors because they feared infection or disliked the experience of consuming certain goods during a pandemic. Households also rebalanced expenditures across time, reducing current expenditures in favor of a future when conditions for consumption are back to normal.

On the other hand, the epidemic also triggered supply shocks that shrank the economy's production possibilities frontier. For example, lock-downs, desire for social distancing in the workplace, and insurance and liability concerns reduced the supply of labor, the capacity with which firms could safely operate, and firms' productivity.

To analyze this situation of divergent sectoral outcomes, we use a general disaggregated model and aggregate up from the micro to the macro level. We allow for an arbitrary number of sectors and factors, as well as unrestricted input-output linkages and elasticities of substitution. We incorporate downward nominal wage rigidities, creditconstraints, and a zero lower bound on nominal interest rates. While the economics of supply and demand shocks are well-understood in one-sector models, this paper provides a comprehensive analysis of these forces in models with multiple sectors and input-output linkages.

In this paper, we provide analytical results on how disaggregated supply and de- 
mand shocks affect output, employment, and inflation. We show that negative sectoral supply shocks are stagflationary, whereas negative demand shocks are deflationary, even though both can cause Keynesian unemployment. ${ }^{1}$ Furthermore, complementarities in production amplify Keynesian spillovers from supply shocks but mitigate them for demand shocks. This means that complementarities reduce the effectiveness of aggregate demand stimulus. We also use a calibrated version of the model to study the Covid19 crisis, decomposing the supply and demand sources of the recession, and answering counterfactual questions about the effects of aggregate demand stimulus. The following paragraphs describe these results and outline the paper in more detail.

After setting up the environment and equilibrium in Section 2, we provide local comparative statics under very general conditions in Section 3, characterizing the response of aggregates such as output, inflation, and unemployment, as well as of disaggregated variables. Whereas both supply and demand shocks reduce output and cause Keynesian unemployment, their effect on the price level is dissimilar. Negative demand shocks are generically deflationary and negative supply shocks are generically inflationary, even in the presence of arbitrarily complicated production networks and highly incomplete markets. This result, which is obvious in a one-sector model, is not immediate in a multi-sector model because supply shocks in one industry can reduce demand in other industries, and in principle, one might imagine that this effect could be so strong as to overturn the intuition from the one-sector model.

Section 3 shows that the production network matters only in so far as it plays a role in determining how factor income shares respond to shocks in equilibrium. To build more intuition for this result, in Section 4, we focus on a Cobb-Douglas special case where the behavior of factor shares is simple to understand. Using the Cobb-Douglas model, we demonstrate that credit-constraints magnify the unemployment and output effects of both supply and demand shocks. If unemployed households are unable to borrow against their future income, then unemployed workers are forced to cut back their spending more aggressively than they would if they could borrow. Therefore, credit-constraints magnify spending reductions given income losses, and this acts as an endogenous negative demand shock.

In Section 5, we consider a CES special case to understand the role of complementarities. We show that complementarities in the production network amplify negative supply

\footnotetext{
${ }^{1}$ Keynesian unemployment measures the amount of slack in a given factor market. It captures underemployment due to lack of demand for the good that the factor is producing because of downwardly rigid wages. Measured unemployment in the data reflects not only Keynesian unemployment but other forms of supply-driven underemployment due to the pandemic. See Section 2.2 for a discussion.
} 
shocks to some markets by causing Keynesian spillovers in other markets. Intuitively, negative supply shocks raise the relative price of the shocked sectors, and because of complementarities, redirect expenditures towards the shocked sectors. This reduces demand in other sectors and causes Keynesian unemployment. In contrast, we show that these complementarities also mitigate demand shocks. In response to a negative demand shock, flexible factor prices fall relative to rigid factor prices. Due to complementarities, expenditures are then redirected away from flexibly-priced factors and towards the Keynesian factor markets, stabilizing employment.

The fact that complementarities mitigate demand shocks is a double-edged sword, since for the same reason, complementarities also mitigate the potency of aggregate demand stimulus. Intuitively, aggregate demand stimulus raises the price of non-Keynesian markets, and substitution towards these markets due to complementarities dissipates the efficacy of stimulus.

In Sections 4 and 5, we also provide global comparative statics showing that the qualitative nature of our local results remain valid for very large shocks. These global comparative statics allow us to capture the nonlinearities of the model and in particular, how the shocks interact with each other and get amplified or mitigated. Under some conditions, we show that output is globally decreasing in negative supply and demand shocks, whereas inflation is globally increasing in negative supply but decreasing in negative demand shocks.

In Section 6, we provide a quantitative illustration of the model applied to US data from February to May, 2020. We use this model to decompose the relative importance of supply and demand shocks, and study the role of complementarities across sectors in shaping the response of output to the initial shocks and to changes in policy.

The benchmark model predicts that real GDP falls by $9 \%$, inflation is $-1 \%$ and there is up to $7 \%$ Keynesian unemployment. Negative supply shocks on their own reduce output by only $6 \%$, cause mild Keynesian unemployment of around $1 \%$, and imply inflation should be close to $7 \%$. On the other hand, negative demand shocks on their own reduce output by $5 \%$, cause $10 \%$ Keynesian unemployment and predict inflation of $-4 \%$. Hence, both supply and demand shocks are necessary to match the data, which features large reductions in real GDP but only mild deflation.

We also use the model to classify sectors as supply-constrained (tight) or demandconstrained (slack). In both the model and the data, supply-constrained sectors experienced mild price inflation, and demand-constrained sectors experienced mild price deflation over our sample period. Furthermore, in the data, wages of workers in sectors the model classifies as being supply-constrained rose whilst those in demand-constrained 
sectors fell.

We also quantify the importance of complementarities. We find that complementarities amplified negative supply shocks and mitigated negative demand shocks by roughly an equal amount, and therefore, do not have strong effects on the overall aggregate response of inflation or output. However, complementarities change the breakdown between the relative importance of supply versus demand, making supply shocks relatively more important.

As mentioned before, an important reason for separating supply-constrained sectors from demand-constrained ones is that they respond very differently to interventions. Demand-side policies are counterproductive in supply-constrained markets, and supplyside policies are unhelpful in demand-constrained markets. In this vein, we consider policy counterfactuals for social insurance and monetary policy.

We find that the power of untargeted aggregate demand stimuli, like monetary policy, is greatly diminished in the current crisis. In our model, aggregate demand stimulus is one quarter as effective in the current crisis compared to a typical, aggregate-demand-driven, recession. There are two reasons for this. First, the sectoral nature of the Covid-19 shock means that around half the labor markets are supply-constrained and do not respond to demand stimulus. Second, realistic complementarities sap the efficacy of demand stimulus by dissipating more of it as inflation.

We also use the model to quantify the importance of social insurance. Our baseline calibration assumes complete markets, and adding credit-constraints further depresses output, inflation, and employment. For example, if 50\% of unemployed workers become credit-constrained and receive no income support from the government, then output falls by an additional 1\%, and Keynesian unemployment increases by an extra $2 \%$. As with monetary policy, the importance of social insurance depends on the strength of complementarities, and in a Cobb-Douglas model with weaker complementarities, social insurance is three times more important.

We end the paper by touching upon some extensions of the basic framework in Section 7 before concluding in Section 8 .

\section{Related Literature}

This paper is part of the literature on economic effects of the Covid-19 crisis, as well as the literature on multi-sector models with nominal rigidities.

Guerrieri et al. (2020) show that negative supply shocks can have negative demand spillovers, under the condition that the intersectoral elasticity of substitution is less than 
the intertemporal one. They also show that this condition is weaker under incomplete markets. Our paper complements theirs by considering both supply and demand shocks in a model with rich input-output linkages. Our results about supply shocks build on and are related to theirs. We show that complementarities in the production network, rather than consumption, can also amplify negative supply shocks, even if the intersectoral and intertemporal elasticities of substitution in consumption are the same. Furthermore, we also show that these supply shocks, despite causing Keynesian unemployment, are nevertheless still inflationary. We also show that while complementarities amplify negative supply shocks, they also mitigate negative demand shocks. For this reason, in our quantitative exercise, a Cobb-Douglas model, without complementarities, predicts almost the same reduction in output and employment as a model with stronger intersectoral complementarities because of these off-setting effects on supply and demand shocks.

Bigio et al. (2020) study optimal policies in response to the Covid-19 crisis in a twosector Keynesian model. We differ in both focus and framework, since we are not focused on optimal policy and instead try to understand the importance of the production structure. $^{2}$ Fornaro and Wolf (2020) study Covid-19 in a New-Keynesian model where the pandemic is assumed to have persistent effects on productive capacity in the future by lowering aggregate productivity growth. The expected loss in future income reduces aggregate demand. They show that a feedback loop can arise between aggregate supply and aggregate demand if productivity growth in turn depends on the level of economic activity. ${ }^{3}$ We differ in that we focus on the effects of current disruptions. Caballero and Simsek (2020) study a different kind of spillover, between asset prices and demand shortages.

Our paper also relates to quantitative multi-sector models. Barrot et al. (2020) study the effect of Covid-19 using a quantitative production network with complementarities and detailed administrative data from France. Bonadio et al. (2020) study the effect of Covid-19 in a quantitive international trade model. Bodenstein et al. (2020) analyze optimal shutdown policies in a two-sector model with complementarities and minimum-scale requirements. Our approach differs from these papers due to our focus on nominal rigidities and Keynesian effects. Brinca et al. (2020) use a statistical model to decompose sectoral outcomes in the Covid-19 crisis into demand- and supply-side sources. Our classification of demand and supply drivers are conceptually different to theirs for reasons we discuss in Section 2. Kaplan et al. (2020) combine an SIR model with a multi-sector heterogeneous

\footnotetext{
${ }^{2}$ Bigio et al. (2020) study a fully dynamic model specified in continuous time, which allows them to analyze how the effects unfold over time.

${ }^{3}$ This could be because of reduced investment in research and development due to a reduced size of the market à la Benigno and Fornaro (2018).
} 
agent New Keynesian model to study the economic impact of the pandemic.

This paper is also related to other work by the authors, especially Baqaee and Farhi (2020b). Whereas in this paper, we study how exogenous shocks interact with nominal frictions and result in involuntary unemployment, Baqaee and Farhi (2020b) is a companion paper where we analyze the nonlinear mapping from changes in hours and household preferences to real GDP. In this companion paper, we find that the negative supply and demand shocks associated with Covid-19 are large enough that accounting for nonlinearities is quantitatively important.

Our analysis is also related to production network models with nominal rigidities, like Baqaee (2015), who studies the effect of targeted fiscal policy and shocks to the sectoral composition of demand in a production network with downward wage rigidity, Pasten et al. (2017) and Pasten et al. (2019) who study propagation of monetary and TFP shocks in models with sticky prices, Ozdagli and Weber (2017) who study the interaction of monetary policy, production networks, and asset prices, and Rubbo (2020) and La'O and Tahbaz-Salehi (2020) who study optimal monetary policy with sticky prices.

\section{Setup}

In this section, we set up the basic model. We describe the problem faced by households and firms, the equilibrium notion, and the shocks that we will be studying.

\subsection{Environment and Equilibrium}

There are two periods, the present denoted without stars, and the future denoted with stars, and there is no investment. ${ }^{4}$ We take the price level in the future as given. As in Krugman (1998) and Eggertsson and Krugman (2012), this is isomorphic to an infinitehorizon model where after an initial unexpected shock in period 1 , the economy returns to a long-run equilibrium with market clearing and full employment. ${ }^{5}$

There is a continuum of households, a set of producers $\mathcal{N}$, and a set of factors $\mathcal{G}$. The economy has the same set of households, producers, and factors in both the present and the future.

\footnotetext{
${ }^{4}$ We abstract from investment in the main body of the paper in order to keep the exposition manageable. We show in Appendix F how our approach generalizes to environments with investment.

${ }^{5}$ Our analysis extends to situations where the crisis lasts for multiple periods without change, as long as we maintain the assumption that there is no investment and no credit constraints; see footnote 13 for more information.
} 
Consumers. There is a continuum of consumers who collectively own all the primary factors. The quantity of factor $f$ supplied is $L_{f} \in[0,1]$. Full employment occurs when $L_{f}=1$ for every $f \in \mathcal{G}$. When the quantity of factor $f$ employed falls, we assume this change comes about via the extensive margin. That is, some fraction $1-L_{f}$ of the owners of factor $f$ become unemployed while the remaining fraction $L_{f}$ continue to receive payment and are fully employed. Some fraction, $1-\phi_{f}$, of the unemployed factor is owned by households that derive their entire income solely from $f$ and cannot borrow against their future income. The remaining fraction $\phi_{f}$ can borrow against their future income. This means that a fraction $\left(1-\phi_{f}\right)$ of the unemployed factor $\left(1-L_{f}\right)$ is owned by households that cannot consume anything in the present and are credit-constrained. We refer to these households, that are unemployed and cannot borrow or consume, as the hand-to-mouth (HtM) households, and we refer to the rest of the households, who are not credit-constrained, as the Ricardian households.

All households have the same intertemporal utility function

$$
(1-\beta) \frac{y^{1-1 / \rho}-1}{1-1 / \rho}+\beta \frac{y_{*}^{1-1 / \rho}-1}{1-1 / \rho}
$$

where $\rho$ is the intertemporal elasticity of substitution (IES), $\beta \in[0,1]$ captures households' time-preferences, and $y$ and $y_{*}$ are current and future consumption. The intertemporal budget constraint for an unconstrained household is

$$
p^{Y} y+\frac{p_{*}^{Y} y_{*}}{1+i}=I+\frac{I_{*}}{1+i^{\prime}}
$$

where $I, I_{*}, p^{Y}$, and $p_{*}^{Y}$ are the income of the household and the price of the consumption good in the present and future, and $(1+i)$ is the nominal interest rate. We omit the HtM households' budget constraint since they simply spend their exogenous future income on the future good and cannot consume in the present.

Now, we turn to the within-period problem. The consumption bundle in the present period is given by

$$
Y=C\left(c_{1}, \ldots, c_{\mathcal{N}} ; \omega_{\mathcal{D}}\right)
$$

a homothetic final-demand aggregator of the final consumptions $c_{i}$ of the different goods $i$. The parameter $\omega_{\mathcal{D}}$ is a preference shifter capturing changes in the sectoral composition of final demand. We normalize shocks to the composition of demand so that, at the initial allocation, they do not directly affect the level of present utility relative to future utility. ${ }^{6}$

\footnotetext{
${ }^{6}$ That is, we assume $C\left(c ; \omega_{\mathcal{D}}\right)=C\left(c ; \omega_{\mathcal{D}}^{\prime}\right)$ where $c$ is the vector of consumption goods the household
} 
Throughout the rest of the paper, we refer to $Y$ as output. ${ }^{7}$

The price $p^{Y}$ of the consumption bundle $Y$ is denoted by

$$
p^{Y}=\mathcal{P}\left(p_{1}, \ldots, p_{\mathcal{N}} ; \omega_{\mathcal{D}}\right) .
$$

where $\mathcal{P}$ is the ideal price index of the quantity index $C$. We also denote by

$$
E=p^{Y} Y
$$

final expenditure in the present period (i.e. nominal GDP). Since the price and quantity of the consumption good in the future is exogenous, we represent these by $\bar{Y}_{*}$ and $\bar{p}_{*}^{Y}$. Future final income and expenditure is then $\bar{E}_{*}=\bar{p}_{*}^{Y} \bar{Y}_{*}$.

Producers. Producer $i$ maximizes profits

$$
\pi_{i}=\max _{\left.y_{i},\left\{x_{i j}\right\}, L_{i f}\right\}} p_{i} y_{i}-\sum_{j \in \mathcal{N}} p_{j} x_{i j}-\sum_{f \in \mathcal{G}} w_{f} L_{i f}
$$

subject to production function

$$
y_{i}=A_{i} F_{i}\left(\left\{x_{i j}\right\}_{j \in \mathcal{N}^{\prime}}\left\{L_{i f}\right\}_{f \in \mathcal{G}}\right),
$$

where $A_{i}$ is a Hicks-neutral productivity shifter, $y_{i}$ is total output, and $x_{i j}$ and $L_{i f}$ are intermediate and factor inputs used by $i$. Without loss of generality, we assume that $F_{i}$ has constant returns to scale. ${ }^{8}$

Market equilibrium. Market equilibrium for goods is standard. The market for $i$ is in equilibrium if

$$
c_{i}+\sum_{j \in \mathcal{N}} x_{j i}=y_{i}
$$

consumes in the no-shock steady-state. In other words, we normalize the sectoral preference shocks so that, on their own, they do not alter intertemporal decisions.

${ }^{7}$ Changes in $Y$ are, to a first-order, the same as changes in real GDP. To define real GDP, we mimic the chainweighted procedures used by national income accountants. Local changes in real GDP are defined by the Divisia index $d \log Y^{G D P}=\sum_{i \in \mathcal{N}}\left(p_{i} c_{i}\right) /\left(\sum_{j \in \mathcal{N}} p_{j} c_{j}\right) d \log c_{i}$. To a first-order approximation, $d \log Y^{G D P}=d \log Y$. Discrete changes in real GDP are defined by integrating the Divisia index $\Delta \log Y^{G D P}=\int d \log Y^{G D P}$. If there are shocks to the composition of final demand $\omega_{\mathcal{D}}$, then real GDP $\Delta \log Y^{G D P}$ and the consumption bundle $\Delta \log Y$ are only equal up to a first-order approximation, and may not be the same at higher orders of approximation. We return to these issues in the quantitative exercise in Section 6.

${ }^{8}$ Following the replication argument of McKenzie (1959), we can treat every production function as though it has constant returns by adding producer-specific fixed factors to the model. 
Market equilibrium for factors is non-standard, the wages of factors cannot fall below some exogenous lower bound. ${ }^{9}$ We say that factor market $f$ is in equilibrium if the following there conditions hold:

$$
\left(w_{f}-\bar{w}_{f}\right)\left(L_{f}-\bar{L}_{f}\right)=0, \quad \bar{w}_{f} \leq w_{f,}, \quad L_{f} \leq \bar{L}_{f}
$$

where

$$
L_{f}=\sum_{i \in \mathcal{N}} L_{i f}
$$

is the total demand for factor $f$. The parameter $\bar{w}_{f}$ is an exogenous minimum nominal wage. The parameter $\bar{L}_{f} \leq 1$ is the maximum quantity of the factor that can be employed, and it may be less than full employment (full employment is represented by $L_{f}=\bar{L}_{f}=1$ ).

In words, there are two possibilities. One possibility is $w_{f} \geq \bar{w}_{f}$ and employment of the factor is equal to its maximum value $L_{f}=\bar{L}_{f}$. In this case, we say that the market is tight, that it clears, and that it is supply-constrained. The other possibility is that $w_{f}=\bar{w}_{f}$ and employment of the factor is less than its potential $L_{f} \leq \bar{L}_{f}$. We then say that the market is slack, that it does not clear, and that it is demand-constrained. In this case, we call the underemployment $\bar{L}_{f}-L_{f}$ of the factor Keynesian unemployment since it is caused by a lack of demand for the good that the factor is producing given the rigid wage.

We only consider two cases: the case where $\bar{w}_{f}$ is equal to its pre-shock market-clearing value, denoting the set of such factors by $\mathcal{L} \subseteq \mathcal{G}$; and the case where $\bar{w}_{f}=-\infty$, making the wage of $f$ flexible and ensuring the market for $f$ always clears, denoting the set of such factors by $\mathcal{K} \subseteq \mathcal{G}$. For concreteness, we call $\mathcal{L}$ the labor factors and $\mathcal{K}$ the capital factors. Figure 2.1 illustrates the supply and demand curves in the factor markets.

Of course, these are just names, in practice, one may easily imagine that certain capital markets could also be subject to nominal rigidities. This can be a way to model firm failures: imagine firms take out within-period loans to pay for their variable expenses, secured against their capital income. If the firm's capital income declines in nominal terms, then the firm defaults on the loan, exits the market, and its capital becomes unemployed for the rest of the period. We build on this observation further in Appendix $G$, where we formally introduce an extensive margin of firm exit. For the body of the paper, we treat capital markets as being frictionless.

We denote the endogenous set of supply-constrained factor markets by $\mathcal{S} \subseteq \mathcal{G}$. In other words, $f \in \mathcal{S}$ if, and only if, $L_{f}=\bar{L}_{f}$. We denote the endogenous set of demand-constrained factor markets by $\mathcal{D} \subseteq \mathcal{G}$. Hence, $f \in \mathcal{D}$ if, and only if, $w_{f}=\bar{w}_{f}$ and $L_{f}<\bar{L}_{f}$. Of course,

\footnotetext{
${ }^{9}$ In Appendix E, we extend the model to allow for some downward wage flexibility.
} 


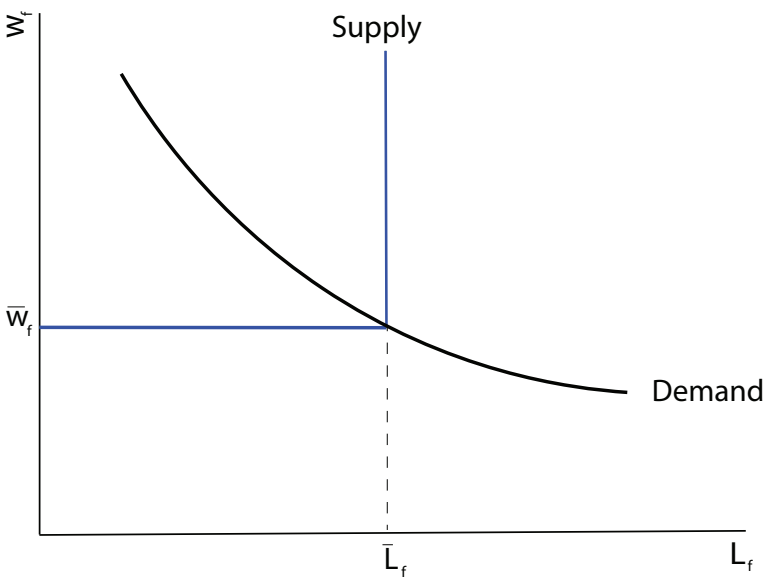

(a) Factors in $\mathcal{L}$

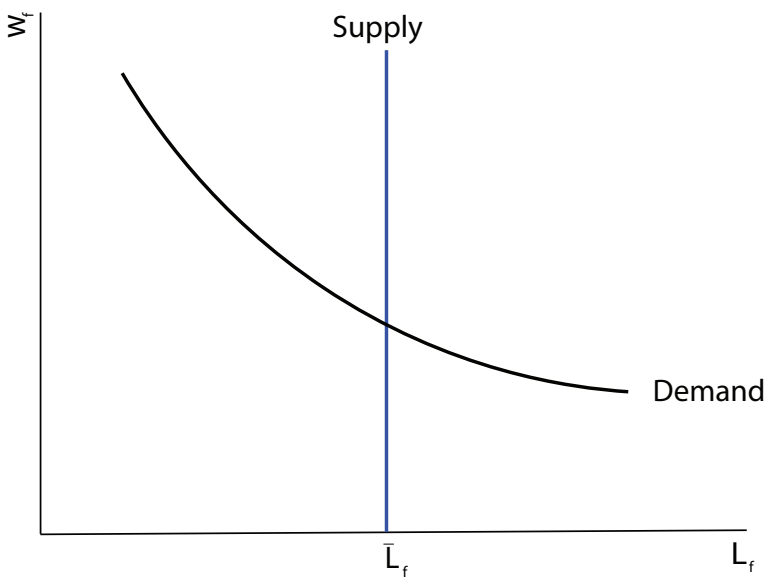

(b) Factors in $\mathcal{K}$

Figure 2.1: Equilibrium in the factor markets.

capital markets are always supply-constrained $\mathcal{K} \subseteq \mathcal{S}$, and demand-constrained sectors are necessarily a subset of labor markets $\mathcal{D} \subseteq \mathcal{L}$.

Equilibrium. Given a nominal interest rate $(1+i)$, future prices $\bar{p}_{*}^{Y}$ and output $\bar{Y}_{*}$, maximum factor supplies $\bar{L}_{f}$, productivities $A_{i}$, and demand shifters $\omega_{\mathcal{D}}$, an equilibrium is a set of prices $p_{i}$, factor wages $w_{f}$, intermediate input choices $x_{i j}$, factor input choices $L_{i f}$, outputs $y_{i}$, and final demands $c_{i}$, such that: each producer maximizes its profits subject to its technological constraint; consumers maximize their utility; and the markets for all goods and factors are in equilibrium. Without loss of generality, we normalize the initial pre-shock values of output and the price level to be one: $\bar{Y}_{*}=Y=p^{Y}=\bar{p}_{*}^{Y}=1$.

\subsection{Supply and Demand Shocks}

Now we define the shocks we study in this paper. A natural disaster, like the Covid-19 epidemic, can be captured as a combination of negative supply and demand shocks. We define supply shocks to be shocks that change the economy's production possibility set. On the other hand, we define a demand shock to be a shock that changes the households' expenditure shares on the different goods (across sectors and over time) at given prices and incomes. We describe each of these shocks in turn.

Supply shocks. Changes in the economy's production possibility set could come in the form of either reduced factors or reduced productivity. We call reductions in the available productive endowment of labor $\bar{L}_{f}$ shocks to potential labor. These are reductions that 
would take place absent any nominal frictions. These reductions could have different drivers. They could be driven directly by government action, like mandated shutdowns and stay-at-home orders. They could also be due to a reduced willingness to work by workers due to fear of infection. Finally, reductions in potential labor could also be the result of a reorganization of production. For example, firms could be forced to operate at lower capacity to reduce legal liability or implement social distancing, such as retailers that can only safely operate at a fraction of their previous capacity. In this case, workers would be involuntarily unemployed due to a reduced physical capacity to employ them and not because there is not enough demand for the good that they produce. ${ }^{10}$ Crucially, these "supply"-driven reductions in employment would occur even in the absence of downward nominal wage rigidities. For this reason, we do not include this form of unemployment in our definition of Keynesian unemployment.

In addition to negative shocks to potential labor, the epidemic might also reduce the productivity $A_{i}$ of the different producers by changing the way firms can operate, for instance by reducing person-to-person interactions.

Demand shocks. Whereas supply shocks change household's choices by changing prices and incomes, demand shocks change household choices for fixed prices and income. ${ }^{11}$ Accordingly, the pandemic can change the current sectoral composition of final demand, since at given prices and income, households may shift expenditure away from some goods like cruises and air transportation, and towards other goods like groceries and online retail. We model this as a change in the preference shifter $\omega_{\mathcal{D}}$.

Similarly, the pandemic can reduce households' willingness to consume in the present relative to the future: at given prices and income, households may choose to consume less during the epidemic and more afterwards. We model this as an increase in the discount

\footnotetext{
${ }^{10}$ To model capacity constraints formally, imagine that $\bar{L}_{f}=\min \left\{1, S_{f}\right\}$, where $S_{f}$ is a "safety" input (capacity) which, in the initial equilibrium, is not scarce. Since it is not scarce, it commands a price of zero initially. However, the pandemic reduces the supply of $S_{f}$ so that it binds. At this point, the supply of potential labor $\bar{L}_{f}$ falls one-for-one with $S_{f}$. In this case, employers would refuse to hire any additional workers since their marginal product is zero. A formal capacity constraint like this is isomorphic to our formulation where we directly shock $\bar{L}_{f}$ in terms of real GDP, inflation, and hours worked. The only difference is that the increase in the wage $w_{f}$ would not take place and would instead be captured as a Ricardian rent by the firm.

${ }^{11}$ Our notion of supply and demand shocks are defined in the context of a general equilibrium, and are not the same as the one used by Brinca et al. (2020). They separate shocks based on whether they shift labor supply or labor demand, but for us, a "supply" shock can shift either labor supply or labor demand. For example, a capacity constraint placed on firms due to social distancing, described in the previous footnote, would manifest as a reduction in labor demand, but be classified as a supply shock under our definition since it reduces the production possibilities of the economy.
} 
factor $\beta /(1-\beta) .{ }^{12}$

\section{General Local Comparative Statics}

In this section, we describe comparative statics of the model that hold regardless of the details of the production side of the economy. Our results here, which are first-order (local) approximations, clarify which sufficient statistics are needed for understanding the responses of output, inflation, and employment to shocks. In Sections 4 and 5, we specialize the model further and write these sufficient statistics in terms of microeconomic primitives. We also provide global (as opposed to first-order) comparative statics in Sections 4 and 5.

Because of downward wage-rigidity, variables like aggregate output and inflation are not differentiable everywhere. Therefore, our local comparative statics should be understood as holding almost-everywhere. Furthermore, there are potentially multiple equilibria, in which case, local comparative statics should be understood as perturbations of a given locally-isolated equilibrium.

We write $d \log X$ for the differential of an endogenous variable $X$ understood as the (infinitesimal) change in an variable $X$ in response to (infinitesimal) shocks. For example, the supply shocks are $d \log A_{i}$ and $d \log \bar{L}_{f}$, and the shocks to the sectoral composition of demand are $d \log \omega_{D}$. For a vector of shocks, like $d \log A$ or $d \log \bar{L}$, we drop the subscripts. For discrete changes in a variable, we write $\Delta \log X$.

Notation. To analyze the model, we introduce some additional notation. Recall that nominal expenditure is the total sum of all final expenditures

$$
E=\sum_{i \in N} p_{i} c_{i}=p^{Y} Y
$$

We define the Domar weight $\lambda_{i}$ of producer $i$ to be its sales share as a fraction of GDP

$$
\lambda_{i} \equiv \frac{p_{i} y_{i}}{E}
$$

The Domar weight $\lambda_{f}$ of factor $f$ is simply its total income share

$$
\lambda_{f} \equiv \frac{w_{f} L_{f}}{E} .
$$

\footnotetext{
${ }^{12}$ In Appendix C, we provide a simple microfoundation for these demand shocks using a health-related disutility function.
} 
Unlike the Domar weight for goods, the Domar weights for factors necessarily sum to one $\sum_{f \in \mathcal{G}} \lambda_{f}=1$. We denote the Domar weight of $f$ in the future by $\lambda_{f}^{*}$.

We proceed in two steps. First, we use the first-order conditions of the household to derive an Euler equation summarizing the intertemporal aspects of the equilibrium. Second, we use the first-order conditions of the firms to derive an aggregation equation that summarizes the intratemporal aspects.

\subsection{Intertemporal Problem}

The consumption of unconstrained households is governed by their Euler equation

$$
y=y_{*}\left[(1+i) \frac{\beta}{1-\beta} \frac{p^{Y}}{\bar{p}_{*}^{Y}}\right]^{-\rho} \text {. }
$$

Since HtM households do not consume in the current period, summing the left-hand side over all non-HtM households yields an expression for aggregate output in the current period

$$
Y=\bar{Y}_{*}\left(1-\sum_{f \in \mathcal{G}} \lambda_{f}^{*}\left(1-\phi_{f}\right)\left(1-L_{f}\right)\right)\left[(1+i) \frac{\beta}{1-\beta} \frac{p^{Y}}{\bar{p}_{*}^{Y}}\right]^{-\rho},
$$

where we use the fact that the share of future output consumed by HtM households is $\sum_{f} \lambda_{f}^{*}\left(1-\phi_{f}\right)\left(1-L_{f}\right)$. Note that the constrained fraction $\left(1-L_{f}\right)\left(1-\phi_{f}\right)$ rises as employment falls, and it does not depend matter whether the reduction in employment in factor market $f$ is due to binding supply or demand constraints. Log-linearizing the Euler equation results in an aggregate demand curve that relates changes in output $d \log Y$ to changes in the price index $d \log p^{Y}$.

Proposition 1 (Intertemporal Optimization). Changes in output are given by

$$
d \log Y=-\rho d \log p^{Y}+d \log \zeta+d \log \Theta,
$$

where $d \log \zeta$ and $d \log \Theta$ are intercepts. The first intercept term is

$$
d \log \zeta=d \log \bar{Y}_{*}-\rho\left(d \log (1+i)+d \log \frac{\beta}{1-\beta}-d \log \bar{p}_{*}^{Y}\right) .
$$

and the second is

$$
d \log \Theta=d \log \left(1-\mathbb{E}_{\lambda^{*}}\left(\left(1-\phi_{f}\right)\left(1-L_{f}\right)\right)\right)
$$


where the expectation uses the factor income shares in the future, $\lambda^{*}$, as the probability distribution.

We call $d \log \zeta$ an aggregate demand shock. A positive aggregate demand shock can come about from an increase in expected future output, reduction in the nominal interest rate or the discount factor, or an increase in future prices (a proxy for forward guidance). ${ }^{13}$

Note that with complete financial markets, $\phi_{f}=1$ for every $f$, the second intercept, $d \log \Theta$, is always zero. We call $d \log \Theta$ the endogenous aggregate demand shock. This term captures the fact that reductions in employment today reduce spending today, since $1-\phi_{f}$ of type $f$ workers become constrained. Therefore, as pointed out by Guerrieri et al. (2020), reductions in employment, $d \log L_{f}<0$, can feed back into reduced nominal demand because some households are hand-to-mouth.

Changes in nominal expenditure $d \log E$ are similarly given by

$$
d \log E=d \log \left(p^{\Upsilon} Y\right)=(1-\rho) d \log p^{\Upsilon}+d \log \zeta+d \log \Theta .
$$

Recall that $\rho$ is the intertemporal elasticity of substitution (IES). When $\rho>1$, increases in prices $d \log p^{Y}>0$ reduce nominal expenditure as consumers substitute towards the future. Conversely, when $\rho<1$, increases in prices $d \log p^{Y}>0$ increase nominal expenditure as consumers substitute towards the present. When $\rho=1$, and there are no HtM households, changes in nominal expenditure are exogenously given by the shocks $d \log E=d \log \zeta$. In this paper, we often focus on the case where $\rho=1$, which is a focal point for the empirical literature on the IES.

\subsection{Intratemporal Problem}

Whereas Proposition 1 is a consequence of the consumers' first-order conditions, the next proposition is a consequence of the producers' first-order conditions and resource constraints.

\footnotetext{
${ }^{13}$ If the crisis lasts for more than one period, and there are no credit-constraints, the Euler equation can still be used to write output in each period as a function of the price index in that period and exogenous shocks. That is, $\Delta \log Y_{t}=-\rho \Delta \log p_{t}^{Y}-\rho\left(\sum_{j=1}^{T} \Delta \log \left(1+i_{t+j-1}\right)+\Delta \log \frac{\beta_{*}}{\beta_{t}}-\Delta \log \bar{p}_{*}^{Y}\right)+\Delta \log \bar{Y}_{*}+d \log \Theta$, where $t$ indexes time and $*$ is the terminal period when the economy recovers. Since this is the only dynamic relationship, the rest of the analysis can be combined with this Euler equation instead to determine output in each period before recovery. This approach is only tenable if the periods are short-lived however, since we assume that the nominal wage constraint is exogenous.
} 
Proposition 2 (Intratemporal Optimization). Changes in output are given by

$$
\begin{aligned}
d \log Y & =\sum_{i \in \mathcal{N}} \lambda_{i} d \log A_{i}+\sum_{f \in \mathcal{G}} \lambda_{f} d \log L_{f}, \\
& =\underbrace{\sum_{i \in \mathcal{N}} \lambda_{i} d \log A_{i}+\sum_{f \in \mathcal{G}} \lambda_{f} d \log \bar{L}_{f}}_{\Delta \text { potential output }}+\underbrace{\sum_{f \in \mathcal{L}} \lambda_{f} \min \left\{d \log \lambda_{f}+d \log E-d \log \bar{L}_{f}, 0\right\} .}_{\Delta \text { output gap }}
\end{aligned}
$$

Equation (3.5) for $d \log Y$ shows that a version of Hulten's (1978) theorem holds for this economy. In particular, to a first-order, changes in output can only be driven by changes in the productivities $d \log A_{i}$ weighted by their producer's sales share $\lambda_{i}$, or by changes in the quantities of factors $d \log L_{f}$ weighted by their income shares $\lambda_{f} .{ }^{14}$

To arrive at Equation (3.6), use the fact that changes in capitals $f \in \mathcal{K}$ are exogenous with

$$
d \log L_{f}=d \log \bar{L}_{f}
$$

and changes in labors $f \in \mathcal{L}$ are endogenous with

$$
d \log L_{f}=\min \left\{d \log \lambda_{f}+d \log E, d \log \bar{L}_{f}\right\} \leq d \log \bar{L}_{f} .
$$

Here we have used the observation that factor $f$ is demand-constrained, with $d \log w_{f}=0$ and $d \log L_{f}=d \log \lambda_{f}+d \log E$ if, and only if, changes in nominal expenditure on this factor $d \log \lambda_{f}+d \log E$ are below changes in its potential supply $d \log \bar{L}_{f}$.

The first term in (3.6) is the change in potential output and corresponds to the change in output that would occur in a neoclassical version of the model with flexible wages. The second term is the negative output gap that can open up in the Keynesian version of the model with downward nominal wage rigidities because of Keynesian unemployment in the different factor markets. These Keynesian spillovers depend on endogenous changes in nominal expenditure $d \log E$, pinned down by the Euler equation (3.4), and factor income shares $d \log \lambda_{f}$ (to be determined in later sections).

Crucially, Proposition 2 clarifies how the details of the production network matter in this economy. It is only through the determination of changes in factor shares, $d \log \lambda_{f}$, that the details of the production network will matter.

\footnotetext{
${ }^{14}$ This expression also shows that changes in the sectoral composition of demand within the period $d \log \omega_{\mathcal{D}}$, or changes in aggregate demand $d \log \zeta$, can only change output through changes in the quantities of factors.
} 
Combining the intra- and intertemporal problems. Without delving into the details of the production network and the disaggregated model, we can already make an observation about the way inflation responds to shocks by combining the intertemporal and intratemporal sides of the model.

Corollary 1 (Inflation). At the full-equilibrium steady-state, the change in the price level is given by

$$
d \log p^{Y}=\frac{1}{\rho} d \log \zeta-\frac{1}{\rho} \sum_{f \in \mathcal{G}} \lambda_{f} \phi_{f} d \log L_{f}-\frac{1}{\rho} \sum_{i \in \mathcal{N}} \lambda_{i} d \log A_{i} .
$$

Hence, reductions in employment, $d \log L<0$, and productivity, $d \log A<0$, are stagflationary unless they are also accompanied by exogenous negative aggregate demand shocks $d \log \zeta<0$. Note that it does not matter whether the reductions in employment $d \log L_{f}$ are supply-driven or demand-driven, either way, they are inflationary. Furthermore, Corollary 1 also shows that shocks to the sectoral composition of demand, $d \log \omega_{\mathcal{D}} \neq 0$, generically raise inflation even though they may cause Keynesian unemployment.

Corollary 1 is remarkably general, since it holds regardless of the disaggregated details of the production structure. Furthermore, it also does not rely on the assumption that some factor wages cannot fall. In particular, in Appendix E, we show that Corollary 1 holds even when wages are fully or semi-flexible. Corollary 1 shows that, in order to model a sharp recession without significant inflation, like the Covid-19 crisis, we must allow for negative aggregate demand shocks.

To prove this corollary, combine Propositions 1 and 2 to get

$$
d \log p^{Y}=\frac{1}{\rho} d \log \zeta+\frac{1}{\rho} d \log \Theta-\frac{1}{\rho} \sum_{i \in \mathcal{N}} \lambda_{i} d \log A_{i}-\frac{1}{\rho} \sum_{f \in \mathcal{G}} \lambda_{f} d \log L_{f}
$$

This equation shows that reductions in $\mathrm{AD}$-shifters, $d \log \zeta$ and $d \log \Theta$, lower the price, whereas reductions in supply, $d \log A$ and $d \log L$, tend to raise the price of the consumption good. While $d \log \zeta$ is exogenous, $d \log \Theta$ is endogenous. At the full-equilibrium steadystate,

$$
d \log \Theta=\sum_{f \in \mathcal{G}} \lambda_{f}^{*}\left(1-\phi_{f}\right) d \log L_{f} .
$$

That is, the endogenous demand shifter depends on the reduction in employment in the current period weighted by the income share of HtM workers in the future. Since at the initial steady-state current and future factor income shares are the same, $\lambda_{f}^{*}=\lambda_{f}$, we can substitute this equation for $d \log \Theta$ into (3.9) to get the desired result. 


\subsection{Input-Output Notation}

Propositions 1 and 2 show that the response of output, inflation, and employment depend on equilibrium changes in factor income shares $d \log \lambda_{f}$. These are the only endogenous objects left to be determined, and it is through these objects that the production network exerts an influence on the outcome variables of interest. ${ }^{15}$ We provide a characterization of changes in factor shares in Appendix D for general networks. In the body of the paper, we specialize the results in Appendix D and focus on a Cobb-Douglas and CES special case. To do so, we define some input-output notation used throughout the rest of the paper.

Input-output and Leontief matrix. We slightly abuse notation by treating factors with the same notation as goods. For each factor $f$, we interchangeably use the notation $L_{i f}$ or $x_{i(\mathcal{N}+f)}$ to denote its use by $i$, the notation $L_{f}$ or $y_{f}$ to denote total factor supply, and $p_{f}$ or $w_{f}$ to refer to its price or wage. Furthermore, we treat final demand as an additional good produced by producer 0 using the final demand aggregator. We interchangeably use $c_{i}$ or $x_{0 i}$ to denote final consumption of good $i$. We write $1+\mathcal{N}$ for the union of the sets $\{0\}$ and $\mathcal{N}$, and $1+\mathcal{N}+\mathcal{G}$ for the union of the sets $\{0\}, \mathcal{N}$, and $\mathcal{G}$. With this abuse of notation, we can stack every market in the economy into a single input-output matrix that includes the household, the producers, and the factors.

The input-output matrix is the $(1+\mathcal{N}+\mathcal{G}) \times(1+\mathcal{N}+\mathcal{G})$ matrix $\Omega$ whose $i j$ th element is equal to $i$ 's expenditures on inputs from $j$ as a share of its total income/revenues

$$
\Omega_{i j} \equiv \frac{p_{j} x_{i j}}{p_{i} y_{i}}=\frac{p_{j} x_{i j}}{\sum_{k \in \mathcal{N}+\mathcal{G}} p_{k} x_{i k}} .
$$

The input-output matrix $\Omega$ records the direct exposures of one producer to another. The first row corresponds to the households' use of inputs, the next $\mathcal{N}$ rows are the producers' uses of inputs, and the last $\mathcal{G}$ rows correspond to the factors's use of inputs (the last $\mathcal{G}$ rows are all equal to zero, since factors do not use any inputs).

The Leontief inverse matrix is

$$
\Psi \equiv(I-\Omega)^{-1}=I+\Omega+\Omega^{2}+\ldots
$$

The Leontief inverse matrix $\Psi$ records instead the direct and indirect exposures through the supply chains in the production network.

\footnotetext{
${ }^{15}$ See Baqaee and Farhi (2021) for assumptions under which the production network is globally irrelevant for comparative statics.
} 
The accounting identity $p_{i} y_{i}=p_{i} x_{0 i}+\sum_{j \in \mathcal{N}} p_{i} x_{j i}=\Omega_{0 i} E+\sum_{j \in \mathcal{N}} \Omega_{j i} \lambda_{j} E$ links the Domar weights to the Leontief inverse via

$$
\lambda_{i}=\Psi_{0 i}=\sum_{j \in \mathcal{N}} \Omega_{0 j} \Psi_{j i}
$$

where $\Omega_{0 j}=\left(p_{j} x_{0 j}\right) /\left(\sum_{k \in \mathcal{N}+\mathcal{G}} p_{k} x_{0 k}\right)=\left(p_{j} c_{j}\right) / E$ is the share of good $j$ in final expenditure. Equation (3.10) is a key equation, showing how the input-output matrix pins down the factor income shares, and through this, affects equilibrium employment and output. Appendix D provides a general characterization of how factor income shares respond to shocks. In the next two sections, we focus on some intuitive parametric special cases instead.

\section{The Cobb-Douglas Economy}

In this section, we study the Cobb-Douglas special case, where intertemporal and intersectoral preferences are log and production functions are Cobb-Douglas. In Section 5, we extend the analysis in this section beyond the Cobb-Douglas special case.

\subsection{Local Comparative Statics}

We analyze the effect of negative supply and demand shocks in turn. Recall that $\mathcal{S}$ and $\mathcal{D}$ are the equilibrium sets of supply- and demand-constrained factors. We give comparative statics for a given $\mathcal{S}$ and $\mathcal{D}$. We then give conditions for these sets of supply- and demandconstrained factors to indeed arise in equilibrium. We start by considering supply shocks. For transparency, we set the share of potentially HtM households in every sector to be the same $\phi_{i}=\phi$.

Supply Shocks. Consider negative factor supply shocks on their own. In response to negative supply shocks, aggregate expenditures fall in the present, since some households are HtM. This reduction in spending reduces employment in demand-constrained factor markets and depresses output further.

To see this, define the average negative labor shock to the supply-constrained factors

$$
d \log \bar{L}_{\mathcal{S}}=\sum_{f \in \mathcal{S}} \frac{\lambda_{f}}{\lambda_{\mathcal{S}}} d \log \bar{L}_{f}
$$


where $\lambda_{\mathcal{S}}=\sum_{f \in \mathcal{S}} \lambda_{f}$. Similarly, the average employment change in the demand-constrained factors is

$$
d \log L_{\mathcal{D}}=\sum_{f \in \mathcal{D}} \frac{\lambda_{f}}{\lambda_{\mathcal{D}}} d \log L_{f}<\sum_{f \in \mathcal{D}} \frac{\lambda_{f}}{\lambda_{\mathcal{D}}} d \log \bar{L}_{f}=d \log \bar{L}_{\mathcal{D}}
$$

where $\lambda_{\mathcal{D}}=\sum_{f \in \mathcal{D}} \lambda_{f}$. Keynesian unemployment is given by $d \log L_{\mathcal{D}}-d \log \bar{L}_{\mathcal{D}}$. Using Proposition 2, we can write

$$
d \log Y=\lambda_{\mathcal{S}} d \log \bar{L}_{\mathcal{S}}+\lambda_{\mathcal{D}} d \log \lambda_{\mathcal{D}}+\lambda_{\mathcal{D}} d \log E=\lambda_{\mathcal{S}} d \log \bar{L}_{\mathcal{S}}+\lambda_{\mathcal{D}} d \log E
$$

The second equality follows from the fact that factor income shares remain constant due to the Cobb-Douglas assumption. Hence, Keynesian unemployment arises if there are reductions in nominal spending $d \log E$.

Using the Euler Equation (3.4), starting at the full employment allocation, the change in nominal spending today is

$$
d \log E=d \log \Theta=(1-\phi) \lambda_{\mathcal{S}} d \log \bar{L}_{\mathcal{S}}+(1-\phi) \lambda_{\mathcal{D}} d \log E=\frac{(1-\phi) \lambda_{\mathcal{S}} d \log \bar{L}_{\mathcal{S}}}{1-(1-\phi) \lambda_{\mathcal{D}}} .
$$

Hence, negative supply shocks reduce nominal spending by reducing the income of credit-constrained consumers directly and indirectly through a Keynesian-cross type effect. Combining these equations results in the following.

Proposition 3 (Supply shocks). Suppose that all within-period production and consumption functions are Cobb-Douglas, $\rho=1$, and $\phi_{j}=\phi$ for all $j \in \mathcal{N}$. Then, in response to negative labor supply shocks $d \log \bar{L}$ we have

$$
d \log Y=\lambda_{\mathcal{S}} d \log \bar{L}_{\mathcal{S}}+\lambda_{\mathcal{D}} d \log L_{\mathcal{D}}=\frac{\lambda_{\mathcal{S}}}{1-(1-\phi) \lambda_{\mathcal{D}}} d \log \bar{L}_{\mathcal{S}}
$$

The direct impact on output of the negative shock to the supply-constrained factors is given by $\lambda_{\mathcal{S}} d \log \bar{L}_{\mathcal{S}}$, and the amplification of this shock through Keynesian channels is given by the multiplier $1 /\left[1-(1-\phi)\left(1-\lambda_{\mathcal{S}}\right)\right]$. Naturally, amplification is stronger, the lower is the social insurance parameter $\phi<1$.

We now go back and check that our conjectured set of supply-constrained factors is indeed the equilibrium set of supply-constrained factors. A factor $f$ is demand-constrained in equilibrium if, and only if, $f \in \mathcal{L}$ and

$$
\frac{(1-\phi)}{1-(1-\phi) \lambda_{\mathcal{D}}} \lambda_{\mathcal{S}} d \log \bar{L}_{\mathcal{S}}<d \log \bar{L}_{f}
$$


That is, as long as the negative shock to factor $f$ is sufficiently small in magnitude compared to the average shock affecting the supply-constrained part of the economy. This condition is harder to satisfy the smaller is the set of supply-constrained factors $\lambda_{\mathcal{S}}$ and the higher is the market completeness parameter $\phi$. In particular, if we assume that there are no creditconstrained households $\phi=1$, then this condition cannot be satisfied and all factors are supply-constrained. In this case, Keynesian frictions would not be triggered in response to supply shocks.

Demand Shocks. When final demand is a Cobb-Douglas aggregator, we can model sectoral demand shocks as a decline in that sector's Cobb-Douglas weight (leaving the other Cobb-Douglas weights unchanged). That is, we assume that within-period utility is given by

$$
\log C=\sum_{i \in \mathcal{N}}\left(\bar{\Omega}_{0 i}-\kappa_{i}\right) \log c_{i}
$$

where $\bar{\Omega}_{0 i}$ is households' initial budget share on good $i$ with $\overline{\mathcal{K}}_{i}=0$ in the initial equilibrium. A decline in demand for $i, \kappa_{i}>0$, maps to shocks to both the intersectoral and intertemporal composition of demand. In particular, the shock changes the composition of demand within the period by

$$
\Delta \log \Omega_{0 i}=\Delta \log \frac{\bar{\Omega}_{0 i}-\kappa_{i}}{\left(1-\sum_{j \in \mathcal{N}} \kappa_{j}\right) \bar{\Omega}_{0 i}},
$$

and it changes the composition of demand across periods according to

$$
\Delta \log \zeta=-\Delta \log (1+i)-\Delta \log \frac{\beta}{1-\beta}+\Delta \log \bar{E}_{*}+\Delta \log \left(1-\sum_{j \in \mathcal{N}} \kappa_{j}\right) .
$$

For future reference, when we refer to an aggregate demand shock, we mean a change in $\Delta \log \zeta$ that keeps the intersectoral composition of final demand constant, that is $\Delta \log \Omega_{0 i}=$ 0 for every $i$.

To understand demand shocks, starting at the full employment steady-state without supply shocks, we consider an aggregate demand shock first and then sectoral demand shocks.

Proposition 4 (Aggregate demand shocks). Suppose that all within-period production and consumption functions are Cobb-Douglas, $\rho=1$, and $\phi_{j}=\phi$ for all $j \in \mathcal{N}$. For an aggregate demand shock, $d \log \zeta$, the change in output is

$$
d \log Y=\lambda_{\mathcal{D}} d \log L_{\mathcal{D}}=\lambda_{\mathcal{D}} d \log E=\frac{\lambda_{\mathcal{D}}}{1-(1-\phi) \lambda_{\mathcal{D}}} d \log \zeta .
$$


Hence, as long as there are some HtM households $\phi \neq 1$, aggregate demand shocks are also amplified by a multiplier $1 /\left(1-(1-\phi) \lambda_{\mathcal{D}}\right)$, for similar reasons to supply shocks. So far, the production network has not mattered for either sectoral supply shocks nor aggregate demand shocks, because these shocks do not change factor income shares.

Finally, consider a vector of sectoral demand shocks $d \kappa$, starting at the full employment steady-state without supply shocks. In this case, reduced demand for good $i$ will ripple up the supply chain and differentially affect different factor markets. To see this, note that in demand-constrained sectors, employment falls according to the reduction in nominal spending

$$
d \log L_{f}=d \log \lambda_{f}+d \log E=d \log \left(\sum_{j} \Psi_{j f}\left(\bar{\Omega}_{0 j}-\kappa_{j}\right)\right)+d \log \Theta,
$$

where the second equality uses the Euler equation for expenditures (3.4). Intuitively, there are two reasons why nominal spending on factor $i$ can fall. First, as emphasized in Baqaee (2015), a negative demand shock $d \kappa_{j}>0$ to consumption good $j$ affects demand for factor $f$ by j's network-adjusted factor intensity $\Psi_{j f}>0$. Intuitively, $\Psi_{j f}$ is the fraction of $j$ 's revenues that are ultimately paid out to factor $f$, both directly and indirectly. This is the first summand. The second summand captures the fact that demand shocks to any demand-constrained factor depresses the income of credit-constrained consumers, and through this, lowers overall expenditures. The equation above is a linear system in $d \log L$, so solving through gives

$$
d \log L_{f}=\frac{-\sum_{j} \Psi_{j f} d \kappa_{j}}{\lambda_{f}\left(1-\sum_{h} \kappa_{h}\right)}-\left[\frac{1-\phi}{\phi} \sum_{g \in \mathcal{D}} L_{g} \frac{\sum_{j} \Psi_{j g} d \kappa_{j}}{\lambda_{i}\left(1-\sum_{h} \kappa_{h}\right)}\right],
$$

the first summand is the direct effect of the negative demand shock and the second summand is the negative spillovers from HtM households. In the complete markets case, with $\phi=1$, only the direct effect matters. However, when there are credit-constrained consumers, the indirect effect also matters.

Combining these observations with Proposition 2 allows us to state the following.

Proposition 5 (Sector-specific shocks). Suppose that all within-period production and consumption functions are Cobb-Douglas, $\rho=1$, and $\phi_{j}=\phi$ for all $j \in \mathcal{N}$. Starting at steady-state, 
for sector-specific demand shocks, $d \kappa$, the change in output is

$$
d \log Y=-\left[\sum_{f \in \mathcal{D}} \sum_{j \in \mathcal{N}} \Psi_{j f} d \kappa_{j}+\frac{1-\phi}{\phi} \sum_{f \in \mathcal{D}} \sum_{j \in \mathcal{N}} \Psi_{j f} d \kappa_{j}\right] .
$$

The first term is the direct effect of the negative demand shock and the second term are the Keynesian spillovers from the presence of HtM households. Unlike aggregate demand shocks, the effects of sectoral demand shocks do depend on the shape of the production network, as these shocks propagate up supply chains.

\subsection{Global Comparative Statics}

We now show that the intuitions developed using derivatives are globally valid. In general, the equilibrium of this model may not be unique. However, for the CobbDouglas economy, there is a simple-to-compute unique "best" equilibrium. We provide global comparative statics for this equilibrium. To formalize this, endow $\mathbb{R}^{\mathcal{G}}$ with the partial ordering $x \leq y$ if and only if $x_{f} \leq y_{f}$ for all $f \in \mathcal{G}$. Recall that we use $\Delta$ to denote discrete changes in a variable to distinguish them from infinitesimal local changes denoted by $d$.

Lemma 1 (Ranking equilibria). Suppose that all within-period production and consumption functions are Cobb-Douglas and $\rho=1$. Then there is a unique best equilibrium: for any other equilibrium, $\Delta \log Y$ and $\Delta \log L$ are lower than at the best equilibrium.

Lemma 1 provides a straightforward way to compute this best equilibrium using an algorithm along the lines of Vives (1990) or, more recently, Elliott et al. (2014). ${ }^{16}$ For the best equilibrium, we can conduct global comparative statics for both supply and demand shocks.

Proposition 6 (Global comparative statics). Under the assumptions of Lemma 1, in the best equilibrium, the following holds:

1. Real GDP $\Delta \log Y$ and employment $\Delta \log L$ are increasing and the price level $\Delta \log p^{Y}$ is decreasing in supply shocks $\Delta \log \bar{L}$.

\footnotetext{
${ }^{16}$ We can find the best equilibrium as follows. Solve the model assuming all factor markets are supplyconstrained. If one of the wages is below the minimum, call this market demand-constrained and set its wage equal to its lower bound. Recompute the equilibrium assuming that these factor markets are demandconstrained. Continue in this manner until the wage in every candidate supply-constrained market is above its lower bound.
} 
2. Real GDP $\Delta \log Y$, employment $\Delta \log L$, and the price level $\Delta \log p^{Y}$ are increasing in exogenous aggregate demand shocks $\Delta \log \zeta$.

3. Employment $\Delta \log L$ is decreasing in individual demand shocks $\Delta \kappa_{i}$.

The global comparative static results in Proposition 6 show that the local-comparative static results hold globally. In particular, (1) shows that negative labor shocks in some factor markets raise the overall price level and, if there are HtM households, create Keynesian unemployment in other factor markets. On the other hand, (2) shows that negative aggregate demand shocks, whether driven by policy, expectations about the future, or health concerns can create Keynesian unemployment whilst lowering the overall price level. Finally, (3) shows that individual demand shocks lower employment globally. ${ }^{17}$

\section{Beyond Cobb-Douglas}

In this section, we extend the analysis in Section 4 beyond the Cobb-Douglas special case to understand the role of complementarities. We focus on an especially tractable case where elasticities of substitution in production are not equal to one, but are symmetric and uniform for every producer. We show that complementarities have dissimilar effects on supply and demand shocks: complementarities amplify Keynesian spillovers from supply shocks, but mitigate Keynesian spillovers from demand shocks. This implies that in the presence of negative supply shocks and complementarities, aggregate demand stimulus is less potent than in the Cobb-Douglas case.

To do this, suppose each good $i \in \mathcal{N}$ is produced with the production function

$$
\frac{y_{i}}{\bar{y}_{i}}=\frac{A_{i}}{\bar{A}_{i}}\left(\sum_{j \in \mathcal{N}+\mathcal{G}} \bar{\omega}_{i j}\left(\frac{x_{i j}}{\bar{x}_{i j}}\right)^{\frac{\theta-1}{\theta}}\right)^{\frac{\theta}{\theta-1}},
$$

where $x_{i j}$ are intermediate inputs from $j$ used by $i, \bar{\omega}_{i j}$ is a demand shifter for $i$ 's use of input $j$, and variables with over-lines are normalizing constants. We assume that the elasticity of substitution in production $\theta$ is less than or equal to one. We keep the consumption function Cobb-Douglas, as in Section 4. This special case is likely to be an empirically important one since elasticities of substitution across two-digit sectors in production are

\footnotetext{
${ }^{17}$ For technical reasons, we do not characterize global (non-local) changes in output and inflation when the sectoral composition of final demand changes. This is because when the sectoral composition of final demand changes, changes in real GDP can no longer be measured using $\Delta \log Y$ globally (only locally). See the path-dependence problem discussed in Baqaee and Farhi (2020b).
} 
frequently estimated to be below one, but the ones in consumption are likely close to one (e.g., see Atalay, 2017; Herrendorf et al., 2013).

\subsection{Local Comparative Statics}

As discussed in Section 3, the key sufficient statistics to be solved for are the changes in factor income shares $d \log \lambda$. To do this, we introduce some additional notation. Denote the $f$ th column of the Leontief inverse by $\Psi_{(f)}$. We denote the covariance of two vectors (of size $1+\mathcal{N}+\mathcal{G}$ ) weighted using the household budget shares $\Omega^{(0)}$ by $\operatorname{Cov}_{\Omega^{(0)}}(\cdot, \cdot)$. Similarly, we denote the expectation of a vector using the household budget shares by $\mathbb{E}_{\Omega^{(0)}}[\cdot]$. Using this notation, we can state the following result.

Proposition 7 (Propagation with Complementarities). Let household preferences be CobbDouglas and production functions be given by (5.1). Then changes in factor income shares solve the following linear system

$$
\begin{aligned}
d \log \lambda_{f}=\frac{1}{\theta} \operatorname{Cov}_{\Omega^{(0)}}\left(d \log \Omega^{(0)}, \frac{\Psi_{(f)}}{\lambda_{f}}\right) & -\frac{1-\theta}{\theta} d \log L_{f} \\
& -\frac{1-\theta}{\theta} \sum_{k \in \mathcal{G}} \mathbb{E}_{\Omega^{(0)}}\left(\Psi_{(k)} \frac{\Psi_{(f)}}{\lambda_{f}}\right)\left(d \log \lambda_{k}-d \log L_{k}\right) .
\end{aligned}
$$

almost everywhere, where changes in factor employments are given by

$$
d \log L_{f}=\left\{\begin{array}{cc}
d \log \bar{L}_{f}, & \text { for } f \in \mathcal{K}, \\
\min \left\{d \log \lambda_{f}+d \log E, d \log \bar{L}_{f}\right\}, & \text { for } f \in \mathcal{L} .
\end{array}\right.
$$

Equation (5.2) describes the endogenous changes in factor income shares in equilibrium. When $\theta=1$, we recover the Cobb-Douglas case, where the network structure is irrelevant for supply shocks and aggregate demand shocks. As long as $\theta \neq 1$, the inputoutput matrix now matters for every shock. We describe the intuition for right-hand side of (5.2) term-by-term.

The first line are partial equilibrium demand and supply shocks respectively, where by partial equilibrium, we mean holding fixed the wage of factors relative to nominal GDP (i.e, setting $d \log \lambda_{k}-d \log L_{k}=d \log w_{k}-d \log E=0$ for every $k \in \mathcal{G}$ ). ${ }^{18}$ The second line is the general equilibrium feedback from the fact that wages respond in equilibrium.

The first term on the right-hand side captures how changes in the sectoral composition of household demand $d \log \Omega_{(0)}$ affect the share of income accruing to $f$. If demand

\footnotetext{
${ }^{18}$ This follows from the fact that, by definition, $\lambda_{k}=w_{k} L_{k} / E$.
} 
shifts in favor of goods that use $f$ intensively, then $\operatorname{Cov}_{\Omega^{(0)}}\left(d \log \Omega_{(0)}, \Psi_{(f)}\right)$ is positive and the income share of $f$ increases. The second term captures the fact that an increase in the supply $d \log L_{f}>0$ depresses spending on $f$ if there are complementarities in the production network $(\theta<1)$.

The terms on the second line are the general equilibrium effects and capture how changes in the wages of factors affect spending on $f$. In particular, note that $d \log \lambda_{k}-$ $d \log L_{k}>0$ implies that the wage of factor $k$ is rising faster than nominal GDP $d \log w_{k}-$ $d \log E>0$. In this case, due to complementarities, the increase in the relative price of $k$ redirects spending away from $f$ and towards $k$. The strength of this general equilibrium effect depends on the similarity of $k$ and $f^{\prime}$ s demand-chain as measured by $\mathbb{E}_{\Omega^{(0)}}\left(\Psi_{(k)} \Psi_{(f)}\right) \geq$ 0 .

To understand why $\mathbb{E}_{\Omega^{(0)}}\left(\Psi_{(k)} \Psi_{(f)}\right)$ measures similarity of demand, note that it is the dot product of two non-negative vectors $\Psi_{(k)}$ and $\Psi_{(f)}$. These two vectors capture the network-adjusted reliance of each good in the economy on factor $k$ and $f$ respectively. The inner product of these two vectors is proportional to the cosine of the angle between them. Intuitively, when $\mathbb{E}_{\Omega^{(0)}}\left(\Psi_{(k)} \Psi_{(f)}\right)$ is large and positive, this means that producers who are heavily reliant on $k$ are also heavily reliant on $f$, and hence, an increase in the price of $k$ will, in the presence of complementarities, reduce $f^{\prime}$ 's share of income. On the other hand, when $\Psi_{(f)}$ and $\Psi_{(k)}$ orthogonal, which happens when $f$ and $k$ have disjoint demand-chains, the shock to the price of $k$ has no direct effect on the income share of $f$.

Proposition 7 together with Propositions 1 and 2 pin down all the endogenous variables in the model in terms of primitives. In Appendix D, we generalize Proposition 7 for production networks with arbitrary elasticities of substitution and nesting structures but the intuition remains similar.

Amplification of Supply and Mitigation of Demand Shocks. To better understand the intuition for why complementarities amplify supply shocks and mitigate demand shocks, consider the following worked-out example.

Proposition 8 (Supply and demand shocks with complementarities). Let household preferences be Cobb-Douglas and production functions be given by (5.1). Consider labor supply shocks $d \log \bar{L}$, aggregate demand shocks $d \log \zeta$, and shocks to the sectoral composition of demand 
$d \log \Omega^{(0)}$. Suppose only one factor is supply-constrained. Then we have

$$
\begin{aligned}
d \log Y & =\left[\lambda_{\mathcal{S}}+\frac{(1-\theta) \mathbb{E}_{\Omega^{(0)}}\left(\Psi_{(\mathcal{D})} \Psi_{(S)}\right) \lambda_{\mathcal{S}}}{\lambda_{\mathcal{S}}-(1-\theta) \mathbb{E}_{\Omega^{(0)}}\left(\Psi_{(\mathcal{D})} \Psi_{(S)}\right)}\right] d \log \bar{L}_{\mathcal{S}} \\
& +\left[\lambda_{\mathcal{D}}-\frac{(1-\theta) \mathbb{E}_{\Omega^{(0)}}\left(\Psi_{(\mathcal{D})} \Psi_{(\mathcal{S})}\right) \lambda_{\mathcal{S}}}{\lambda_{\mathcal{S}}-(1-\theta) \mathbb{E}_{\Omega^{(0)}}\left(\Psi_{(\mathcal{D})} \Psi_{(S)}\right)}\right] d \log E+\frac{\lambda_{\mathcal{S}} \operatorname{Cov}_{\Omega^{(0)}}\left(d \log \Omega^{(0)}, \Psi_{(\mathcal{D})}\right)}{\lambda_{\mathcal{S}}-(1-\theta) \mathbb{E}_{\Omega^{(0)}}\left(\Psi_{(\mathcal{D})} \Psi_{(S)}\right)},
\end{aligned}
$$

where $\lambda_{\mathcal{D}}=\sum_{f \in \mathcal{D}} \lambda_{f}=1-\lambda_{\mathcal{S}}$, and $\Psi_{\mathcal{D}}=\sum_{f \in \mathcal{D}} \Psi_{(f)}$. When there is full social insurance ( $\phi_{f}=1$ for every $f \in \mathcal{G}$ ), changes in nominal GDP are equal to aggregate demand shocks $d \log E=d \log \zeta$.

The first line is the effect of supply shocks $\left(d \log \bar{L}_{\mathcal{S}}\right)$, and the second line is the effect of aggregate or sector-specific demand shocks $\left(d \log E\right.$ and $\left.d \log \Omega_{(0)}\right)$. We consider the intuition for supply shocks first and then consider the intuition for demand shocks. For this discussion, note that $0<\lambda_{\mathcal{S}}-(1-\theta) \mathbb{E}_{\Omega^{(0)}}\left(\Psi_{(\mathcal{D})} \Psi_{(\mathcal{S})}\right)<1 .{ }^{19}$

The first line shows that a negative supply shock to the supply-constrained factor, $d \log \bar{L}_{\mathcal{S}}$, reduces output directly by the income share of the factor $\lambda_{\mathcal{S}} d \log \bar{L}_{\mathcal{S}}$ and indirectly due to complementarities. When supply-chains between the supply-constrained factor $\mathcal{S}$ and the demand constrained factors $\mathcal{D}$ are similar, the supply shock causes expenditures to switch away from $\mathcal{D}$ and towards $\mathcal{S}$, causing Keynesian unemployment and further reducing output. Therefore, unlike the Cobb-Douglas model, complementarities amplify the effects of the negative supply shock, even in the absence of credit-constraints.

Now consider the effect of shocks to aggregate demand $d \log E=d \log \zeta+d \log \Theta$. As explained, these negative demand shocks may be exogenous $d \log \zeta$ or endogenous $d \log \Theta$. Either way, reduced nominal spending reduces output directly by $\lambda_{\mathcal{D}} d \log E<0$ since it reduces employment in demand-constrained sectors one-for-one. However, this effect is mitigated by complementarities. When nominal spending falls $d \log E<0$ this reduces the price of the supply-constrained factor relative to the demand-constrained factors, redirecting spending away from the supply-constrained factor and stabilizing employment in the demand-constrained factor markets. This means that complementarities mitigate aggregate demand shocks because shocks that change nominal spending $d \log E$ are dissipated by complementarities.

The final summand on the second line of Equation (5.3) captures the effect of shocks to the sectoral composition of demand. Unlike aggregate demand shocks, shocks to the sectoral composition of demand are magnified by complementarities. Intuitively, a sectoral demand shock that moves final demand away from demand-constrained sectors, captured

\footnotetext{
${ }^{19}$ This follows from the fact that $\lambda_{\mathcal{S}}=\mathbb{E}_{\Omega^{(0)}}\left(\Psi_{(\mathcal{S})}\right), 0 \leq \theta<1$, and the fact that $\Psi_{(\mathcal{D})}=1-\Psi_{(\mathcal{S})}$.
} 
by $\operatorname{Cov}_{\Omega^{(0)}}\left(d \log \Omega_{(0)}, \Psi_{(\mathcal{D})}\right)<0$, causes the price of supply-constrained sectors to rise because it redirects that demand towards supply-constrained factors $\operatorname{Cov}_{\Omega^{(0)}}\left(d \log \Omega_{(0)}, \Psi_{(\mathcal{S})}\right)=$ $-\operatorname{Cov}_{\Omega^{(0)}}\left(d \log \Omega_{(0)}, \Psi_{(\mathcal{D})}\right)>0$. In the presence of complementarities, this increase in price reinforces the substitution away from demand-constrained sectors. ${ }^{20}$

\subsection{Global Comparative Statics}

Proposition 8 takes the set of demand- and supply-constrained factor markets as given. We now generalize the global comparative static results to account for the fact that these sets are determined endogenously. As in Section 4, we begin by proving that the set of equilibria can still be ranked.

Lemma 2 (Ranking equilibria with complementarities). Suppose that household preferences are Cobb-Douglas across sectors, $\rho=1$, and the elasticity of substitution in production is $\theta<1$. Then there is a unique best equilibrium: for any other equilibrium, $\Delta \log Y$ and $\Delta \log L$ are lower than at the best equilibrium.

The following proposition shows that the qualitative insights from the Cobb-Douglas economy continue to hold.

Proposition 9 (Global comparative statics with complementarities). Under the assumptions of Lemma 2, in the best equilibrium, the comparative statics in Proposition 6 still hold.

Therefore, although complementarities change the quantitative behavior of the model, the qualitative predictions are unchanged relative to the Cobb-Douglas model.

\section{Quantitative Illustration}

We now turn to a quantitative illustration. We use a parsimonious and highly stylized quantitative model to disentangle supply and demand shocks and conduct counterfactuals. We calibrate our model to match the peak to trough reductions in employment from February, 2020 to May, 2020. We show that complementarities amplify supply shocks and mitigate demand shocks to roughly off-setting effects. We also show that social insurance is crucial for ameliorating the effects of the crisis, significantly raising output, prices, and

\footnotetext{
${ }^{20}$ For completeness, shocks to the households' Cobb-Douglas shares $d \kappa$, which are a mixture of intertemporal and intratemporal demand shocks, affect output by $d \log Y=-\sum_{i}\left(\Psi_{i \mathcal{D}}-\frac{(1-\theta)\left(1-\Psi_{i \mathcal{D})} \mathbb{E}_{\Omega^{(0)}}\left(\Psi_{(\mathcal{S})} \Psi_{(\mathcal{D})}\right)\right.}{\lambda_{\mathcal{S}}-(1-\theta) \mathbb{E}_{\Omega^{(0)}}\left(\Psi_{(\mathcal{S})} \Psi_{(\mathcal{D})}\right)}\right) d \kappa_{i}$. The intuition for these is very similar to that of aggregate demand shocks.
} 
employment. Finally, we show that the sectorally disparate nature of the Covid-19 crisis, coupled with complementarities, has significantly sapped the potency of aggregate demand stimulus compared to a traditional demand-driven recession.

\subsection{Calibration}

We start by describing how we calibrate the model's parameters and then describe how we calibrate the demand and supply shocks.

Calibrating model parameters. Our quantitative model has 66 industries and industrial production functions are nested-CES aggregators of labor, capital, and intermediates. We set the elasticity of substitution between labor and capital to 0.5 , between value-added and intermediate inputs to 0.6 , across intermediates to 0.2 . We assume that household demand is Cobb-Douglas across goods within each period, and we set the intertemporal elasticity of substitution $\rho=1.0$. These elasticities are broadly in line with estimates from Atalay (2017), Herrendorf et al. (2013), Oberfield (2013), and Boehm et al. (2019), and close to our theoretical benchmark in Section 5 .

The share parameters of utility functions and production functions are calibrated so that at the initial pre-shock allocation, expenditure shares match those in the 2015 annual U.S.input-output tables the BEA once we drop the government, non-comparable imports, and second-hand scrap industries. We focus on the short run and assume, following Baqaee and Farhi (2019a), that labor and capital cannot be reallocated across sectors.

We assume that sectoral labor markets feature perfectly rigid downward nominal wages, but suppose that sectoral capital markets have perfectly flexible rental rates. Goods prices are also set competitively and flexibly. Finally, since personal incomes did not decline during our sample, due to large government transfer programs, we assume full social insurance and set the fraction of households that become HtM to zero for the initial calibration. We consider counterfactuals with imperfect social insurance at the end of the section.

We now describe how we calibrate the primitive demand and supply shocks.

Calibrating demand shocks. Since both the intertemporal and intersectoral elasticities of substitution are equal to one for the household, realized changes in household spending patterns can be directly fed into the model as demand shocks (because household expenditure shares do not depend on relative prices). Our data for realized changes in spending come from the BEA. Using equation (3.4), we calibrate the discount factor shock 
to deliver a $-9.5 \%$ reduction in nominal GDP to match the reduction in nominal GDP between the first and second quarter of 2020. We calibrate shocks to the sectoral composition of demand to match the sectoral composition of personal consumption expenditures in May, 2020. Since personal consumption is about $2 / 3$ of final demand, we downweight shocks to the sectoral composition of demand by $2 / 3$. This is equivalent to assuming that sectoral composition of other components of final demand has not changed (principally, this is private investment and government spending). The left panel of Figure A.1 in Appendix A shows the demand shocks by sector.

Calibrating supply shocks. We assume that supply shocks only affect the quantity of potential labor used by each industry. Given the demand shocks, and taking the structure of the model literally and assuming there is no labor mobility across sectors, we can use the vector of changes in hours by sector as the primitive supply shocks. This is because if a labor market is supply-constrained, then the only way to match hours in that market is via a reduction in potential employment. On the other hand, if a labor market is demand-constrained and has Keynesian unemployment, then any reduction in potential labor supplied up to the realized reduction in hours will have no effect on any observed outcome. ${ }^{21}$ This also means that supply shocks in demand-constrained sectors are not identified, since the resource constraint is not binding in those markets.

In reporting our results, we resolve this ambiguity by setting supply shocks in demandconstrained sectors to zero. This choice does not matter for our baseline results in terms of aggregate and sectoral output, inflation, and employment but it does maximize the amount of Keynesian unemployment we measure. We do this because the other extreme, where we minimize the amount of Keynesian unemployment is uninteresting and results in zero Keynesian unemployment. This is because we can always imagine that there were negative supply shocks in demand-constrained sectors that were exactly equal to the observed reduction in hours in that sector.

We calibrate the primitive supply shocks to match changes in hours worked by sector from the May, 2020 BLS Economic News release following the procedure described in Baqaee et al. (2020). The right panel of Figure A.1 in Appendix A shows the sectoral

\footnotetext{
${ }^{21}$ This results in good fit to employment data. The (size-weighted) average industry-level error in hours in non-healthcare sectors is $2.3 \%$. Our simulations predict counterfactually large reductions in employment by hospitals and ambulatory health care services. However, despite large reductions in expenditures on these sectors (from reduced elective procedures, etc.), in the data, healthcare industries do not show large reductions in employment. Presumably, this reflects the fact that the excess capacity in the healthcare industry is not wasted. Healthcare workers are instead engaged in non-market activities related to the pandemic. Due to the unique role these sectors play in the pandemic, we exclude them here.
} 
supply.

\subsection{Out-of-Sample Fit}

Having calibrated the model and the shocks, we now discuss the model's predictions about variables that we did not use for calibration. We judge the model's performance in terms of aggregate output, prices, and wages. At the aggregate level, the model predicts a reduction of real GDP of around $-8.9 \%$ and a reduction in inflation of around $-0.6 \%$, which are both in line with the decline in real GDP and deflation measured by the BEA for our sample period. ${ }^{22}$ Since we did not target changes in aggregate inflation or real GDP in our calibration, the model does well in terms of matching the aggregates.

At a more disaggregated level, we compare the change in industry-level prices in the model to realized changes in producer prices over the sample period. In the model, demand-constrained sectors experience $-5.3 \%$ inflation and supply-constrained sectors experience inflation of $1.0 \%$. In the data, those sectors that are demand-constrained (according to the model) experienced inflation of $-2.4 \%$ whereas those identified by the model as being supply-constrained had inflation of 1.0\%. Figure A. 2 in Appendix A shows a scatter plot of prices in the model against the data at the sectoral level, and Tables 1 and 2 report the list of demand- and supply-constrained industries along with the observed and model-implied price changes.

Overall, the model performs a reasonable job of predicting price changes, despite being highly stylized. The model does somewhat overpredict the magnitude of disaggregated price changes. This may be due to the fact that some capital markets also have nominal rigidities, ${ }^{23}$ goods prices are also likely to be sticky, and producers may be unwilling to raise prices during a crisis, which are issues we have abstracted away from.

We also consider the model's performance in terms of matching changes in wages. We construct a measure for hourly industry-level wages by combining information from the Quarterly Census on Employment and Wages (QCEW) with the Current Population Survey (CPS). The QCEW reports industry-level average weekly wages, defined as the total weekly wage bill for an industry divided by the number of employees. The CPS reports weekly hours per worker for workers in different industries. We compute changes in hourly wages by subtracting the change in average weekly hours from the CPS (between

\footnotetext{
${ }^{22}$ We measure real GDP and the change in inflation using chained Tornqvist approximations to the Divisia index along a linear path.

${ }^{23}$ As explained in Section 2, downward price rigidity in capital markets can be justified by appealing to nominal rigidities in credit markets, where firms whose nominal capital income falls violate financial covenants, default on their loans, and their capital becomes unemployed for the rest of the period.
} 
February and May of 2020) from changes in weekly wages (between the second and first quarter of 2020) from the QCEW.

The (wage-bill weighted) average wage inflation in supply-constrained sectors in the data is $2.0 \%$ and in demand-constrained industries is $-7.9 \%$. In other words, the sectors that the model identifies as being supply-constrained experienced wage inflation whereas those the model identifies as demand-constrained experienced significant wage deflation. This is especially interesting since hours fell by more in supply-constrained sectors ($17 \%)$ than in demand-constrained sectors $(-11 \%)$. Therefore, supply-constrained sectors reduced hours by more than demand-constrained sectors, and yet experienced wage inflation, which is highly suggestive that these industries were affected by supply constraints. As with prices, since no wage data is used to calibrate the model, the large difference in wage inflation in the two sets of industries indicates that the model is able to separate supply and demand constraints.

Despite this success, comparing wages in the data and the model is problematic for two reasons. First, if the within-industry composition of workers changes towards lower-paid workers, then measured industry-level wages fall by more than true wages. Changing job composition is a major barrier to detecting downward wage rigidity in aggregated data like ours (see, for example, Hazell and Taska, 2020). ${ }^{24}$

Second, the change in wages in supply-constrained sectors is not uniquely pinned down in our model (see Footnote 10 for formal details). This is because reductions in potential labor are isomorphic to reductions in production capacity. For output, employment, and price inflation, reductions in capacity and potential labor are equivalent, but the implications for wages are different. If the supply shocks are entirely due to capacity constraints, then the increases in wages in supply-constrained sectors do not take place and are instead captured as Ricardian rents by the producers. In other words, the change in wages in supply-constrained sectors depends on what fraction of the increase in rent windfalls is captured by workers relative to firms. Thus, we can only provide a range of possible wage changes in supply-constrained industries, and any number between $0 \%$ and $19 \%$ is consistent with the model. In the demand-constrained sectors, wage inflation is of course necessarily $0 \%$ because of the binding downward wage rigidity. 

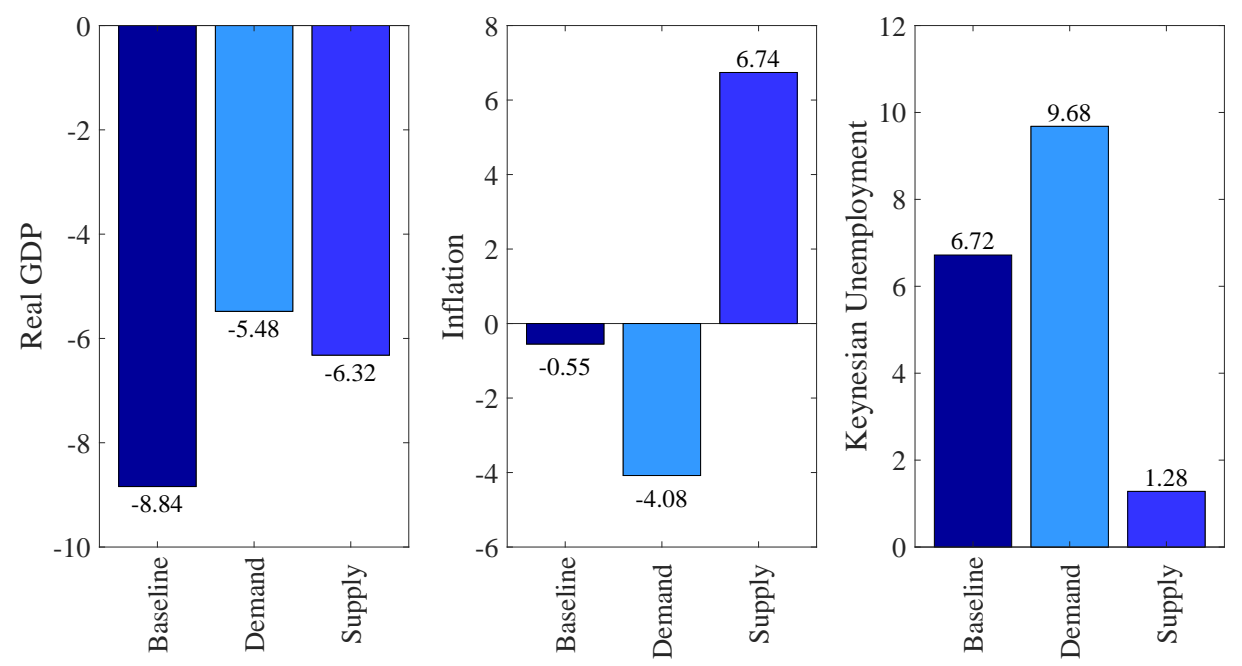

Figure 6.1: Real GDP, inflation, and Keynesian unemployment as a function of shocks for the model with complementarities. The "Baseline" line includes negative demand and supply shocks. The "Supply" bar only includes the sectoral supply shocks. The "Demand" bar only includes the demand shocks.

\subsection{Importance of Supply and Demand Shocks}

Having discussed the model's out-of-sample fit to the data, we now use the model to decompose the importance of supply and demand shocks. Figure 6.1 displays the baseline calibration and decomposes the effect into only supply or only demand shocks. The "Baseline" is the model which includes both the negative demand and negative sectoral supply shocks. The "Supply" bar features only the negative sectoral supply shocks whereas the "Demand" bar features only the demand shocks.

As mentioned before, supply shocks in demand-constrained sectors are not pointidentified, since the resource constraint is not binding in those markets. In other words, there could be negative supply shocks in demand-constrained sectors that are unobservable since these sectors are not operating at capacity. In reporting our results, we resolve this ambiguity by setting supply shocks in demand-constrained sectors to zero, which means that we pick the minimum-sized supply shocks that are consistent with the model. Of course, in practice, demand-constrained sectors also likely experienced negative demand shocks, however, since these shocks are unobservable in our model, we choose the calibration that allows us to best illustrate the economic forces our theoretical analysis highlights. Given this choice, we discuss the decomposition for real GDP, prices, and unemployment in turn.

\footnotetext{
${ }^{24}$ In practice, wages are also not perfectly rigid downwards. Allowing for this is not conceptually difficult, see Appendix E for a description of how the model can be extended to cover semi-flexible wages.
} 
Real GDP. Figure 6.1 shows that negative demand shocks lead to a 5.5\% reduction of real GDP and negative supply shocks reduce real GDP by $6.3 \%$. Because of nonlinearities, the effect of the shocks together $(-8.9 \%)$ is not the same as the sum of the two shocks. Intuitively, reductions in demand in sectors already experiencing large negative supply shocks do not reduce output by as much.

Prices. Although the supply shocks on their own generate large reductions in output, Figure 6.1 shows that they also generate very substantial amounts of inflation around $7 \%$. Meanwhile, the demand shocks, on their own, generate substantial deflation of around $4 \%$. The baseline model, on the other hand, predicts an inflation rate of around $-0.6 \%$. The baseline model performs relatively well, since most price indices show either moderate inflation or moderate deflation. For instance, CPI inflation for this period was $-0.9 \%$ while PCE inflation was $-0.7 \%{ }^{25}$ Both supply and demand shocks are needed to make sense of the large output reduction and moderate deflation observed in the data.

Unemployment. We measure Keynesian unemployment by the reduction in hours in labor markets that are demand-constrained. ${ }^{26}$ As mentioned above, this means that we assume that demand-constrained sectors received no negative supply shocks and hence, Figure 6.1 is the maximum amount of Keynesian unemployment consistent with the model.

Figure 6.1 shows that the negative demand shocks, on their own, generate about $9.7 \%$ Keynesian unemployment. The "Supply" bar in the figure shows that sectoral supply shocks, on their own, generate $1.3 \%$ Keynesian unemployment. Since this calibration has complete markets, this amplification effect is entirely due to complementarities, as discussed in Section 5.1. Together, the supply and demand shocks generate around 7\% Keynesian unemployment, which is less than demand shocks on their own, since some of the sectors hit with negative demand shocks are supply-constrained once we account for the negative supply shocks.

\footnotetext{
${ }^{25}$ The PCE is computed as a Fisher index and it therefore has changing weights reflecting the changing sectoral composition of final demand (unlike the CPI) and is therefore consistent with our model. On the other hand, the PCE does not capture changes in product variety, which could be of concern during lockdowns. Jaravel and $\mathrm{O}^{\prime}$ Connell (2020) show that disappearing goods increased the effective inflation rate in the UK by around 80 basis points. This bias is not large enough to significantly affect our conclusions. We refer the reader to Section $G$ for an extension of the model which allows for disappearing varieties.

${ }^{26}$ Keynesian unemployment is defined as $\sum_{f \in \mathcal{L}}\left(\bar{\lambda}_{f} / \bar{\lambda}_{\mathcal{L}}\right)\left(\Delta \log \bar{L}_{f}-\Delta \log L_{f}\right) \geq 0$, where $\bar{\lambda}_{\mathcal{L}}=\sum_{f \in \mathcal{L}} \bar{\lambda}_{f}$. This captures the percentage underutilization of efficiency units of labor across labor markets.
} 


\subsection{Tightness and Slackness Across Sectors}

Although almost all sectors experienced reductions in hours, in some sectors, these reductions are due to supply constraints whilst in others they are due to demand shortfalls (see Figure A.3 for a complete description). In the baseline, 30 factor markets are demandconstrained and 36 factor markets are supply-constrained.

Supply-constrained sectors include food products and beverages $(-8 \%)$, food services and accommodations $(-39 \%)$, construction $(-9 \%)$, and motion pictures $(-54 \%)$. We interpret the reduction in hours in these sectors to be driven by state-mandated lockdowns, social distancing orders that limited capacity, and employers' fears of being held legally liable should their employees get sick. These restrictions and fears were severe during March and early April. Recall that supply-constrained does not necessarily imply that the reductions are driven by reductions in labor supply or workers' willingness to work. Rather, a supply-constrained sector is one where an increase in nominal demand for the good the sector produces would not translate into increased employment.

Demand-constrained sectors include transportation industries, like air transportation $(-40 \%)$, water transportation $(-43 \%)$, rail transportation $(-19 \%)$, and petroleum and coal $(-21 \%)$ and oil and gas extraction $(-18 \%) .{ }^{27}$ These are industries which experienced sharp reductions in nominal spending, either directly by the household, or indirectly through the supply chain.

\subsection{Role of Complementarities}

Figure 6.2 displays aggregate outcomes in a version of the model where we set all elasticities of substitution equal to one - that is, the Cobb-Douglas model in Section 4.

Real GDP, inflation, and unemployment. In the Cobb-Douglas model, real GDP declines by around $8 \%$ in response to the shocks, which is similar to the response of the benchmark model. However, the breakdown between supply and demand is quite different. The supply shocks, on their own, reduce real GDP by only $4.8 \%$ (compared to $6.3 \%$ in the benchmark) while the demand shocks reduce real GDP by $5.9 \%$ (compared to $5.5 \%$ in the benchmark). Hence, as explained in Section 5.1, complementarities amplify the importance of supply shocks and mitigate the effect of demand shocks, and these effects

\footnotetext{
${ }^{27}$ Our simulations also show that healthcare related industries, like hospitals and ambulatory health care services also experienced reductions in employment of $(-19 \%)$ and $(-16 \%)$. However, presumably, this excess capacity in the healthcare industry is not wasted but engaged in non-market activities related to the pandemic.
} 

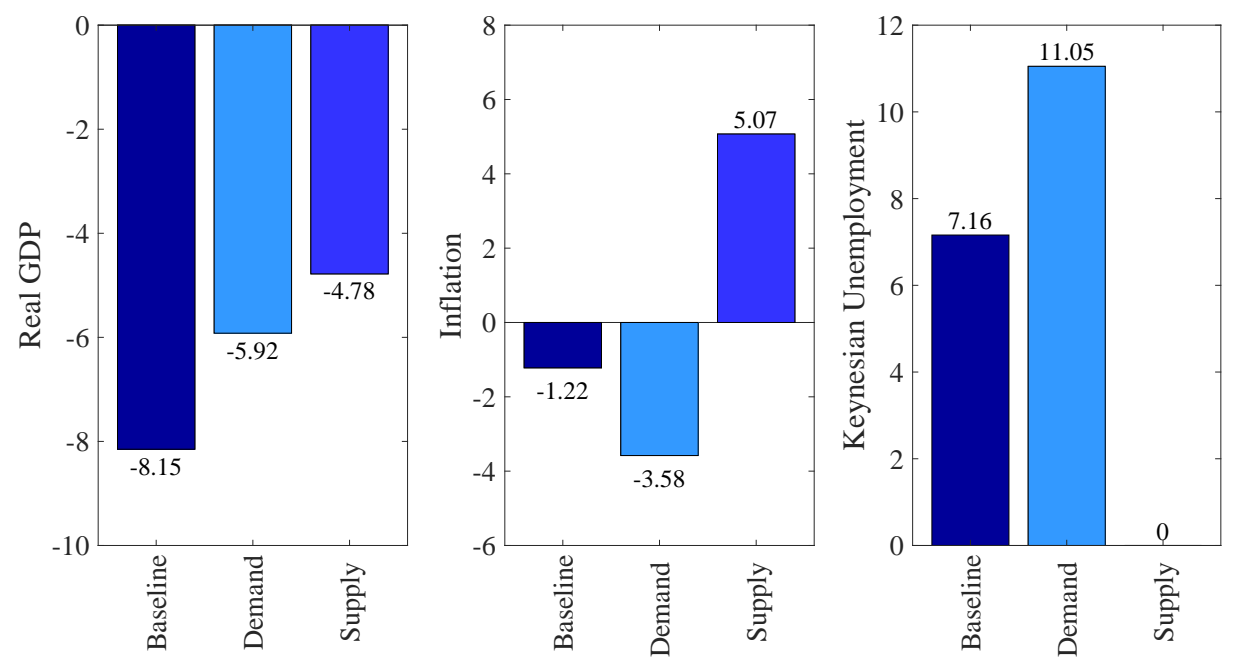

Figure 6.2: Real GDP, inflation, and Keynesian unemployment as a function of shocks without complementarities. The "Baseline" line includes negative demand and supply shocks. The "Supply" bar only includes the sectoral supply shocks. The "Demand" bar only includes the demand shocks.

seem to be roughly off-setting one another.

With only sectoral supply shocks, Keynesian unemployment is now $0 \%$ (instead of $1.3 \%$ in the benchmark). This follows from the discussion in Section 4: this version of the model has complete markets and no complementarities, so supply shocks in one sector do not change nominal spending on other sectors, and hence do not have Keynesian spillovers.

\subsection{Policy Implications}

We end this section by considering some policy counterfactuals. Two important policy tools used to combat adverse effects of the Covid-19 pandemic have been stimulative monetary policy and increased social insurance, in the form of transfers like unemployment benefits. We discuss both of these in turn.

Implications for aggregate demand management. Sectorally disparate supply and demand shocks blunt the power of aggregate demand stimulus. Conventional monetary policy, forward guidance, and untargeted fiscal policy boost aggregate demand. However, with heterogeneous supply and demand shocks, reversing the decline in aggregate demand is not enough to offset the negative effect of the shocks.

To see this, we consider the reduction in real GDP in response to a pure negative demand shock, holding fixed the sectoral composition of final demand and setting sup- 
ply shocks to zero. In the Cobb-Douglas model, the negative aggregate demand shock associated with Covid-19, on its own, reduced real GDP by around 5\%. Therefore, a large enough aggregate demand stimulus can raise real GDP by around $5 \%$ fully offsetting the negative aggregate demand shock. However, with the full set of supply and demand shocks, the same sized aggregate demand stimulus raises real GDP from $-8.2 \%$ to $-5.8 \%$. In other words, the same aggregate demand stimulus only raises real GDP by around $2.2 \%$. Hence, the presence of sectoral shocks cuts the potency of aggregate demand stimulus by half in the Cobb-Douglas model.

In the model with complementarities, this effect is even more extreme. Whereas the aggregate demand shock on its own reduces output by $3.9 \%$, with the full set of sectoral supply and demand shocks, reversing the reduction in aggregate demand through stimulus only boosts output by around $1.1 \%$. Hence, the potency of the aggregate demand stimulus is cut almost by a factor of four in the model with complementarities. Intuitively, this is because the increase in aggregate demand raises the price of supply-constrained factors, and complementarities then cause expenditures to switch towards these factors and away from demand-constrained ones. This reduces the stimulative effect of aggregate demand stimulus.

If we think of the model without sectoral shocks as a typical recession, this means that aggregate demand stimulus is significantly less effective in the Covid-19 recession than in a typical recession. The reason is that without sectoral shocks, the reduction in aggregate demand renders all labor markets demand constrained, and starting from there, an increase in aggregate demand increases employment in all labor markets. By contrast, with sectoral shocks, some labor markets are supply constrained, and starting from there, an increase in aggregate demand is partly dissipated in wage increases in supply-constrained labor markets (the more so, the stronger the complementarities across sectors).

Reduced Social Insurance. Figure 6.3 shows how aggregate outcomes change in the model with complementarities and in the Cobb-Douglas model as we vary the share of households that are potentially HtM. As expected, the presence of HtM households amplifies the reduction in real GDP, reduces inflation, and causes Keynesian unemployment. For example, in the Cobb-Douglas model, when all unemployed workers are HtM, real GDP falls by $13 \%$ rather than $8 \%$, with very significant deflation of $-8 \%$ rather than $-1 \%$, and Keynesian unemployment of $15 \%$ rather than $7 \%$. This underscores the important role that transfers have played in mitigating the negative demand effects associated with the Covid-19 crisis. In the absence of these policies, employment and output would be 
significantly lower.

These numbers are smaller with complementarities, since the endogenous negative aggregate demand shock associated with HtM households is partially absorbed by supplyconstrained factor markets. Specifically, in response to the negative endogenous aggregate demand shock, the price of capital and supply-constrained labor declines, which triggers substitution away from supply-constrained markets towards demand-constrained markets due to complementarities. This is a quantitatively significant stabilizing force in the model. Nevertheless, even in the model with complementarities, social insurance is still very important.

Inflation, the middle panel of Figure 6.3, is relatively insensitive to complementarities. This is to be expected when there are complete markets, because in this case, the complementarities amplify the supply shocks by roughly the same amount that they mitigate the demand shocks, leaving the overall change in real GDP (and hence the price level) roughly unchanged. This is also to be expected at the opposite extreme when all unemployed workers are credit-constrained. According to Corollary 1, when all unemployed workers are $\mathrm{HtM}, \phi_{f}=0$, the change in the price level is just the negative aggregate demand shock $d \log p^{Y}=d \log \zeta \approx-9 \%$ regardless of complementarities.

Intuitively, when markets are incomplete, reductions in employment in the CobbDouglas model are greater (and so inflation is higher), but these reductions are less and less inflationary as we lower $\phi$ because, for lower $\phi$, reductions in employment also reduce total nominal expenditures and hence raise inflation by less. At the extreme point where $\phi=0$, reductions in employment cease to be inflationary.
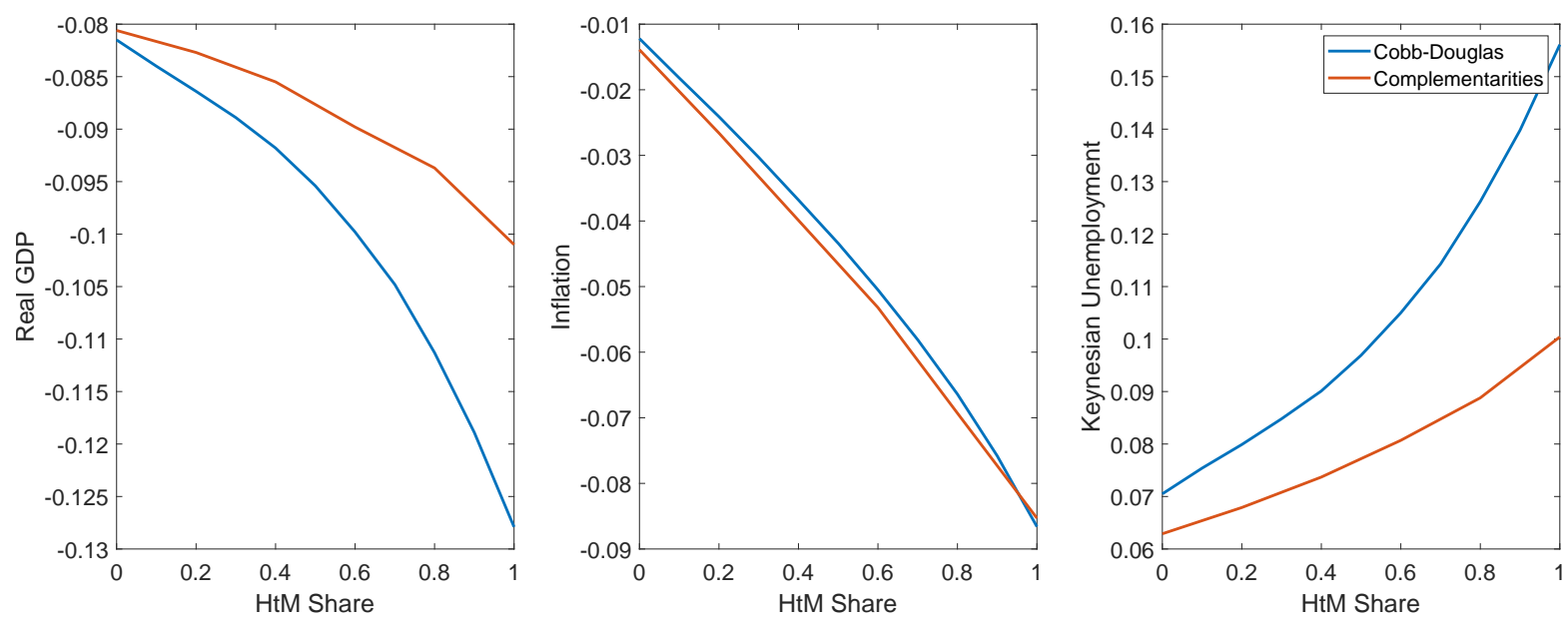

Figure 6.3: Real GDP, inflation, and Keynesian unemployment in the Cobb-Douglas model and the model with complementarities as a function of the share of potentially HtM workers. 


\section{Extensions}

In this section, we briefly summarize extensions of the basic framework that appear in the appendix. Appendix D provides local comparative statics for production networks with arbitrary elasticities of substitution. Appendix E extends our results to the case where wages are semi-flexible. Appendix $G$ extends the framework to cover capital market imperfections and bankruptcies. In this appendix, we show that firm exits act like endogenous negative productivity shocks. Accordingly, they are amplified by inputoutput linkages (just as exogenous productivity shocks are amplified by input-output linkages). Furthermore, exits change relative prices, and these relative price changes can

redirect the flow of spending and cause Keynesian spillovers, much as negative supply shocks. Finally, we also show how exits can result in scarring effects since firms that exit today may not be replaced in the future, this lowers output in the future, which reduces aggregate demand today via the Euler equation (a mechanism emphasized by Fornaro and Wolf, 2020). Appendix F generalizes the results in Section 3 to environments with investment and establishes global comparative statics.

\section{Conclusion}

This paper analytically characterizes the impact of supply and demand shocks in disaggregated economies with multiple sectors, factors, and input-output linkages, as well as occasionally-binding downward nominal wage rigidity, credit-constraints, and a zero lower bound. Using a stylized model, we numerically quantify the impact of supply and demand shocks associated with the Covid-19 crisis, zooming in on the role of complementarities and the implications for aggregate demand management.

Separating the supply and demand sources for the crisis are important since supplyand demand-constrained industries respond differently to policy interventions. Nevertheless, the analysis in this paper is purely positive. For a normative analysis, we would have to take a stance on the health-related externalities of production and consumption. In particular, it may be that implementing the flexible price allocation is not necessarily optimal once we account for these externalities. Nevertheless, the results of any normative analysis would rely on understanding the positive forces analyzed in this paper.

\section{References}

Atalay, Enghin, “How important are sectoral shocks?," American Economic Journal: Macroeconomics, 2017, 9 
(4), 254-80.

Baqaee, David and Emmanuel Farhi, "The Macroeconomic Impact of Microeconomic Shocks: Beyond Hulten's Theorem," Econometrica (Forthcoming), 2019.

_ and _ , "Entry versus Rents," Technical Report 2020.

_ and _ , "Nonlinear Production Networks with an Application to the Covid-19 Crisis," Technical Report 2020.

_ and _ , "Keynesian Production Networks and the Covid-19 Crisis: A Simple Benchmark," Technical Report 2021.

_ , _ , Michael J Mina, and James H Stock, "Reopening scenarios," Technical Report, National Bureau of Economic Research 2020.

Baqaee, David Rezza, “Targeted Fiscal Policy,” 2015.

_ , “Cascading failures in production networks," Econometrica, 2018, 86 (5), 1819-1838.

- and Emmanuel Farhi, "The Microeconomic Foundations of Aggregate Production Functions," Journal of the European Economic Association, 2019, 17 (5), 1337-1392.

Barrot, Jean-Noel, Basile Grassi, and Julien Sauvagnat, "Sectoral effects of social distancing," Available at SSRN, 2020.

Benigno, Gianluca and Luca Fornaro, "Stagnation traps," The Review of Economic Studies, 2018, 85 (3), 1425-1470.

Berman, Abraham and Robert J Plemmons, "Nonnegative matrices," The Mathematical Sciences, Classics in Applied Mathematics,, 1979, 9.

Bigio, Saki, Mengbo Zhang, and Eduardo Zilberman, "Transfers vs Credit Policy," Technical Report 2020.

Bodenstein, Martin, Giancarlo Corsetti, and Luca Guerrieri, "Social Distancing and Supply Disruptions in a Pandemic," 2020.

Boehm, Christoph E, Aaron Flaaen, and Nitya Pandalai-Nayar, "Input linkages and the transmission of shocks: Firm-level evidence from the 2011 Tōhoku earthquake," Review of Economics and Statistics, 2019, 101 (1), 60-75.

Bonadio, Barthélémy, Zhen Huo, Andrei A. Levchenko, and Nitya Pandalai-Nayar, "Global Supply Chains in the Pandemic," Technical Report 2020.

Brinca, Pedro, Joao Duarte, and Miguel Faria e Castro, "Measuring Sectoral Supply and Demand Shocks during COVID-19," Technical Report 2020.

Caballero, Ricardo J and Alp Simsek, "A Model of Asset Price Spirals and Aggregate Demand Amplification of a'COVID-19'Shock," Available at SSRN 3576979, 2020.

Eggertsson, Gauti B and Paul Krugman, "Debt, Deleveraging, and the Liquidity Trap: A Fisher-MinskyKoo Approach," The Quarterly Journal of Economics, 2012, 127 (3), 1469-1513.

Elliott, Matthew, Benjamin Golub, and Matthew O Jackson, "Financial Networks and Contagion," American Economic Review, 2014, 104 (10), 3115-53.

Fornaro, Luca and Martin Wolf, “Covid-19 coronavirus and macroeconomic policy,” 2020.

Guerrieri, Veronica, Guido Lorenzoni, Ludwig Straub, and Iván Werning, "Macroeconomic Implications of COVID-19: Can Negative Supply Shocks Cause Demand Shortages?," Technical Report, National Bureau of Economic Research 2020.

Hazell, Jonathon and Bledi Taska, "Downward Rigidity in the Wage for New Hires," 2020.

Herrendorf, Berthold, Richard Rogerson, and Akos Valentinyi, "Two perspectives on preferences and structural transformation," American Economic Review, 2013, 103 (7), 2752-89. 
Hulten, Charles R, "Growth accounting with intermediate inputs," The Review of Economic Studies, 1978, pp. 511-518.

Jaravel, Xavier and Martin O'Connell, "Inflation Spike and Falling Product Variety during the Great Lockdown," 2020.

Kaplan, Greg, Benjamin Moll, and Gianluca Violante, "Pandemics according to HANK," 2020.

Krugman, Paul R, "It's baaack: Japan's slump and the return of the liquidity trap," Brookings Papers on Economic Activity, 1998, pp. 137-205.

La'O, Jennifer and Alireza Tahbaz-Salehi, "Optimal Monetary Policy in Production Networks," Technical Report 2020.

McKenzie, Lionel W, "On the existence of general equilibrium for a competitive market," Econometrica: journal of the Econometric Society, 1959, pp. 54-71.

Milgrom, Paul and John Roberts, “Comparing equilibria," The American Economic Review, 1994, pp. 441-459.

Oberfield, Ezra, "Productivity and Misallocation During a Crisis: Evidence from the Chilean Crisis of 1982," Review of Economic Dynamics, January 2013, 16 (1), 100-119.

Ozdagli, Ali and Michael Weber, "Monetary policy through production networks: Evidence from the stock market," Technical Report, National Bureau of Economic Research 2017.

Pasten, Ernesto, Raphael Schoenle, and Michael Weber, "Price Rigidity and the Granular Origin of Aggregate Fluctuations," 2017.

$\ldots, \ldots$, and _ , "The propagation of monetary policy shocks in a heterogeneous production economy," Journal of Monetary Economics, 2019.

Rubbo, Elisa, "Networks, Phillips Curves and Monetary Policy," Technical Report 2020.

Tarski, Alfred, "A Lattice Theoretical Fixed Point Theorem and its Applications," Pacific Journal of Mathematics, 1955, 5 (2), 285-309.

Vives, Xavier, "Nash equilibrium with strategic complementarities," Journal of Mathematical Economics, 1990, 19 (3), 305-321. 


\section{Appendix A Additional Graphs and Tables}
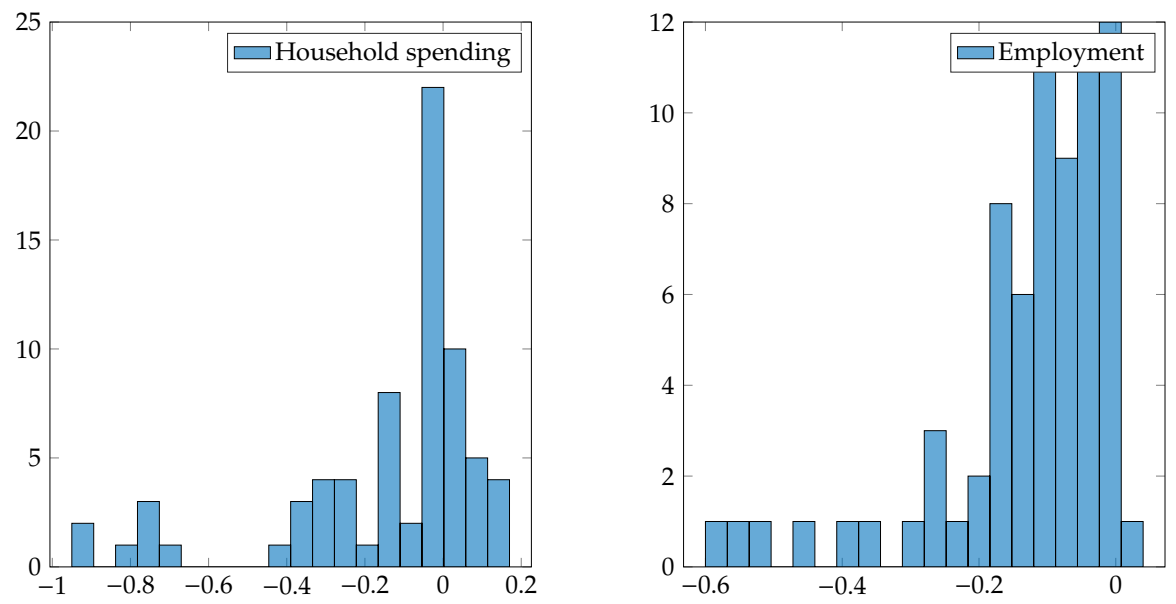

Figure A.1: Reduction in nominal household spending (left panel) and hours worked (right panel) as fractions by sector in May, 2020 compared to February, 2020.

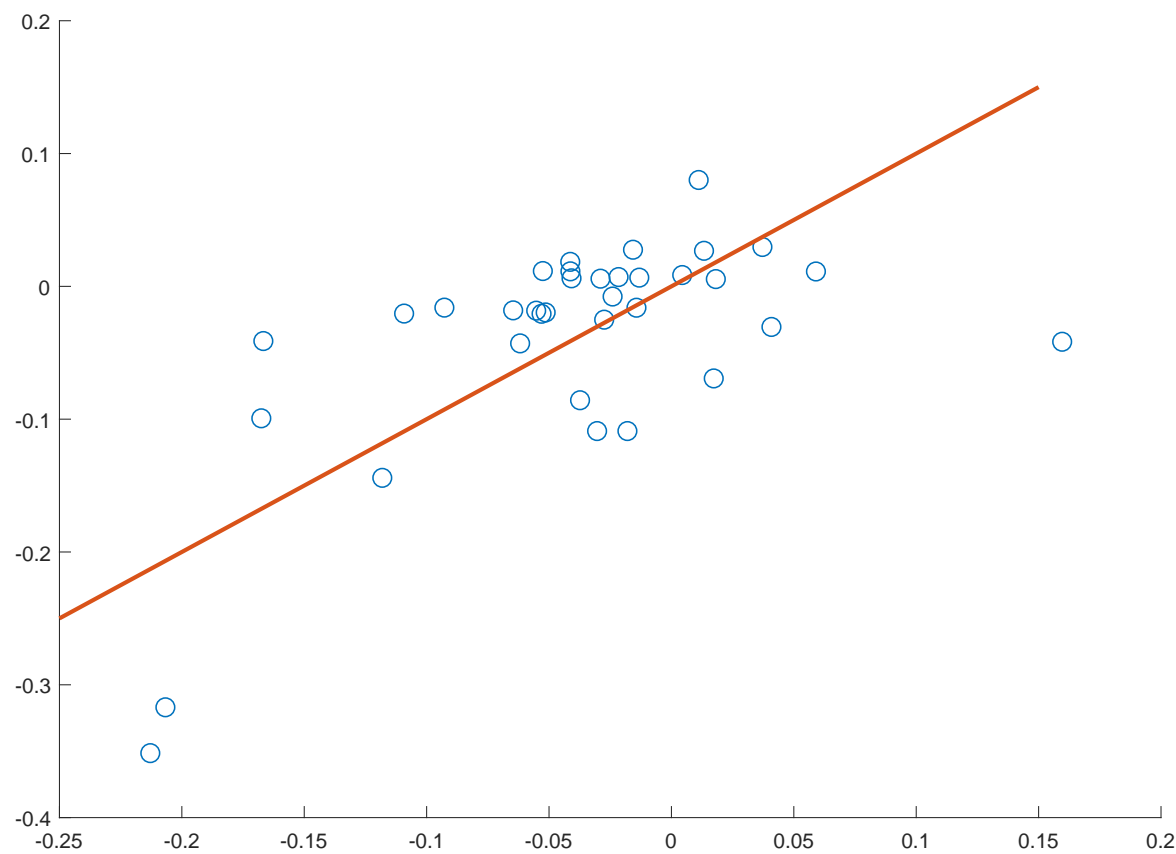

Figure A.2: Changes in model implied prices are on the $\mathrm{x}$-axis and changes in producer prices are on the $y$-axis. The red line is the 45 -degree line. 


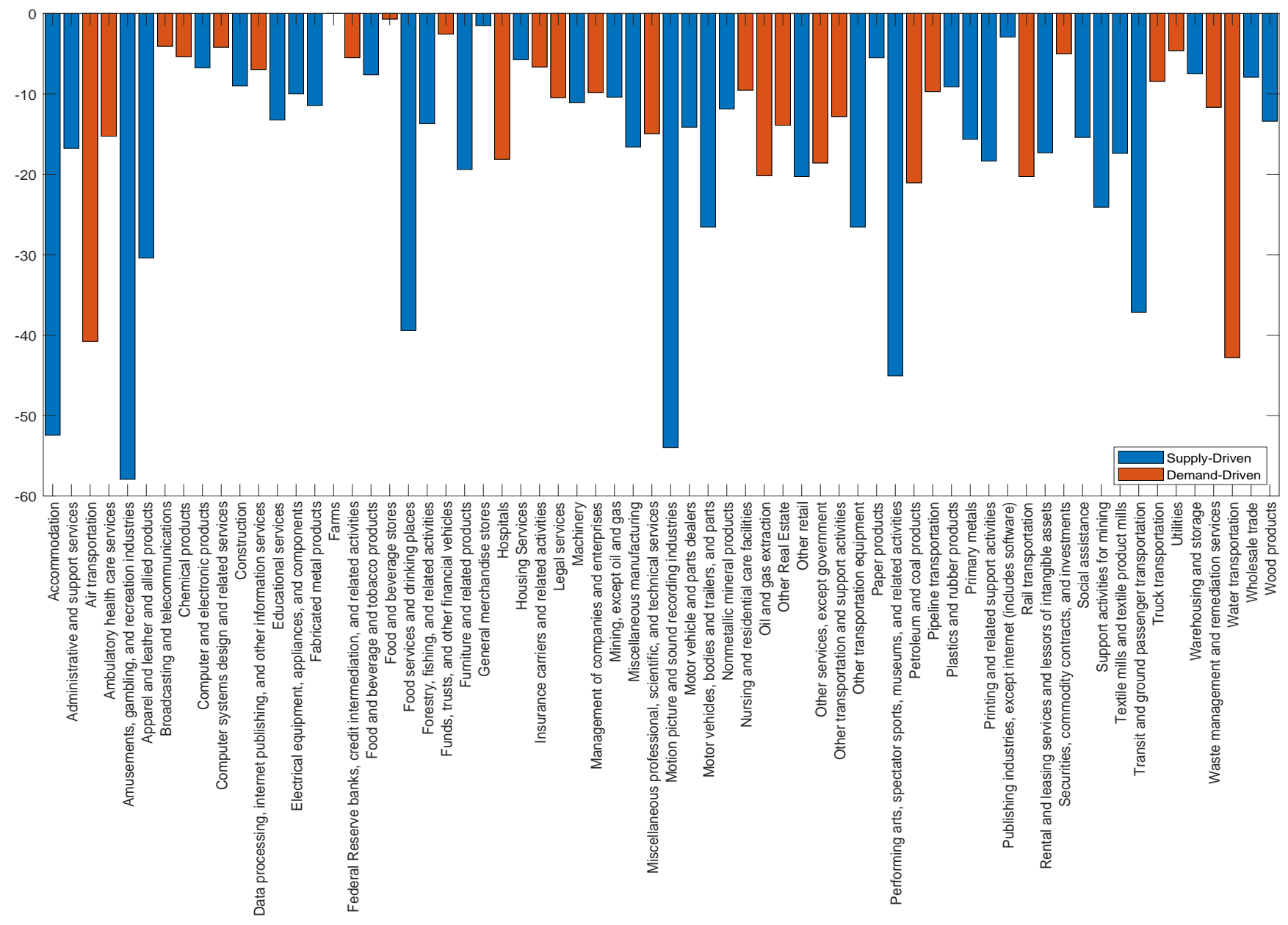

Figure A.3: Model implied percentage reduction in hours by sector from February to May 2020. Sectors below capacity are "demand-driven." 


\begin{tabular}{lrr}
\hline Demand-Constrained Sectors & Inflation (model) & Inflation (data) \\
\hline Petroleum and coal products & -0.21 & -0.35 \\
Oil and gas extraction & -0.20 & -0.33 \\
Air transportation & -0.17 & -0.10 \\
Water transportation & -0.17 & -0.04 \\
Rail transportation & -0.11 & -0.02 \\
Pipeline transportation & -0.10 & -0.01 \\
Utilities & -0.07 & -0.02 \\
Legal services & -0.07 & 0.00 \\
Truck transportation & -0.07 & -0.04 \\
Other transportation and support activities & -0.06 & -0.02 \\
Chemical products & -0.06 & -0.02 \\
Other services, except government & -0.06 & 0.00 \\
Miscellaneous professional/scientific/technical services & -0.06 & -0.01 \\
Waste management and remediation services & -0.05 & 0.01 \\
Insurance carriers and related activities & -0.05 & 0.00 \\
Ambulatory health care services & -0.05 & 0.02 \\
Credit intermediation, related activities & -0.04 & -0.09 \\
Broadcasting and telecommunications & -0.04 & -0.02 \\
Funds, trusts, and other financial vehicles & -0.03 & -0.11 \\
Hospitals & -0.03 & 0.00 \\
Paper products & -0.03 & -0.01 \\
Nursing and residential care facilities & -0.02 & 0.01 \\
General merchandise stores & -0.02 & 0.01 \\
Food and beverage stores & -0.02 & 0.02 \\
Securities, commodity contracts, and investments & -0.02 & -0.11 \\
\hline \hline
\end{tabular}

Table 1: Demand-constrained sectors with model-implied and observed growth rate in prices from February to May, 2020. Sectors with missing prices are excluded. 


\begin{tabular}{lrr}
\hline Supply-Constrained Sectors & Inflation (model) & Inflation (data) \\
\hline Rental and leasing services and lessors of intangible assets & -0.12 & -0.13 \\
Amusements, gambling, and recreation industries & -0.03 & 0.00 \\
Plastics and rubber products & -0.03 & 0.00 \\
Warehousing and storage & -0.03 & 0.00 \\
Mining, except oil and gas & -0.03 & -0.01 \\
Computer and electronic products & -0.02 & 0.00 \\
Nonmetallic mineral products & -0.01 & 0.00 \\
Wholesale trade & -0.01 & 0.01 \\
Food and beverage and tobacco products & 0.00 & 0.08 \\
Miscellaneous manufacturing & 0.01 & 0.00 \\
Administrative and support services & 0.01 & 0.02 \\
Electrical equipment, appliances, and components & 0.01 & 0.00 \\
Construction & 0.01 & 0.00 \\
Accommodation & 0.02 & -0.08 \\
Textile mills and textile product mills & 0.02 & 0.00 \\
Other retail & 0.02 & 0.02 \\
Primary metals & 0.03 & -0.03 \\
Fabricated metal products & 0.03 & 0.00 \\
Publishing industries, except internet (includes software) & 0.04 & 0.00 \\
Machinery & 0.04 & 0.00 \\
Printing and related support activities & 0.05 & 0.00 \\
Wood products & 0.05 & 0.00 \\
Motor vehicle and parts dealers & 0.06 & 0.00 \\
Furniture and related products & 0.07 & 0.00 \\
Support activities for mining & 0.13 & -0.03 \\
Forestry, fishing, and related activities & 0.15 & 0.00 \\
\hline \hline
\end{tabular}

Table 2: Supply-constrained sectors with model-implied and observed growth rate in prices from February to May, 2020. Sectors with missing prices are excluded. 


\section{Appendix B Proofs and Additional Results}

Proof of Proposition 2. By Shephard's lemma, changes in the price of good $i$ are given by

$$
d \log p_{i}=-d \log A_{i}+\sum_{j \in \mathcal{N}} \Omega_{i j} d \log p_{j}+\sum_{f \in \mathcal{G}} \Omega_{i f} d \log w_{f},
$$

solving this system gives

$$
d \log p_{i}=-\sum_{j \in \mathcal{N}} \Psi_{i j} d \log A_{j}+\sum_{f \in \mathcal{G}} \Psi_{i f} d \log w_{f} .
$$

Furthermore,

$$
d \log w_{f}=d \log \lambda_{f}+d \log E-d \log L_{f} .
$$

Hence, the change in real GDP is given by

$$
\begin{aligned}
d \log Y & =d \log E-\sum_{j \in \text { mathcal }} \Omega_{0 j} d \log p_{j}, \\
& =d \log E+\sum_{j \in \mathcal{N}} \Psi_{0 j} d \log A_{j}-\sum_{f \in \mathcal{G}} \Psi_{0 f} d \log w_{f}, \\
& =d \log E+\sum_{j \in \mathcal{N}} \Psi_{0 j} d \log A_{j}-\sum_{f \in \mathcal{G}} \Psi_{0 f}\left(d \log \lambda_{f}+d \log E-d \log L_{f}\right), \\
& =d \log E+\sum_{j \in \mathcal{N}} \lambda_{j} d \log A_{j}-\sum_{f \in \mathcal{G}} \lambda_{f}\left(d \log \lambda_{f}+d \log E-d \log L_{f}\right), \\
& =\sum_{j \in \mathcal{N}} \lambda_{j} d \log A_{j}+\sum_{f \in \mathcal{G}} \lambda_{f} d \log L_{f},
\end{aligned}
$$

using the fact that $\Psi_{0 i}=\lambda_{i}$ and $\sum_{f \in \mathcal{G}} \lambda_{f}=1$. To complete the proof, note that

$$
d \log L_{f}=\min \left\{d \log \bar{L}_{f}, d \log \lambda_{f}+d \log E-d \log \bar{L}_{f}\right\} .
$$

Proof of Proposition 3. The proof is provided in text.

Proof of Proposition 4. This is a special case of Proposition 8.

Proof of Proposition 5. Combine (4.1) and (4.2) with Proposition 8 and let $\theta=1$.

Proof of Lemma 1. This is a special case of Lemma 2. 
Proof of Proposition 6. This is a special case of Proposition 9.

Proof of Proposition 7. From Proposition 10 in Appendix D, we know that

$$
\begin{aligned}
d \log \lambda_{k}=\operatorname{Cov}_{\Omega^{(0)}}\left(d \log \omega_{0}, \frac{\Psi_{(k)}}{\lambda_{k}}\right) \\
+\sum_{j \in \mathcal{N}} \lambda_{j}(\theta-1) \operatorname{Cov}_{\Omega^{(j)}}\left(\sum_{i \in \mathcal{N}} \Psi_{(i)} d \log A_{i}-\sum_{f \in \mathcal{G}} \Psi_{(f)}\left(d \log \lambda_{f}-d \log L_{f}\right), \frac{\Psi_{(k)}}{\lambda_{k}}\right) .
\end{aligned}
$$

with

$$
d \log L_{f}=\left\{\begin{array}{cc}
d \log \bar{L}_{f}, & \text { for } f \in \mathcal{K}, \\
\min \left\{d \log \lambda_{f}+d \log E, d \log \bar{L}_{f}\right\}, & \text { for } f \in \mathcal{L} .
\end{array}\right.
$$

Now, use the identity

$$
\sum \lambda_{j \in 1+\mathcal{N}} \operatorname{Cov}_{\Omega^{(j)}}\left(\Psi_{(f)}, \Psi_{(k)}\right)=\lambda_{k} \lambda_{f}\left[\frac{\Psi_{f k}-\delta_{f k}}{\lambda_{k}}+\frac{\Psi_{k f}-\delta_{f k}}{\lambda_{f}}+\frac{\delta_{f k}}{\lambda_{k}}-1\right]
$$

where $\delta_{f k}$ is Kronecker delta, to get

$$
\begin{aligned}
d \log \lambda_{k} & =\operatorname{Cov}_{\Omega^{(0)}}\left(d \log \omega_{0}, \frac{\Psi_{(k)}}{\lambda_{k}}\right)-(\theta-1) \sum_{f \in \mathcal{G}}\left[\delta_{f k}-\lambda_{f}\right]\left(d \log \lambda_{f}-d \log L_{f}\right) \\
& +\frac{1}{\lambda_{k}} \sum_{f \in \mathcal{G}}(\theta-1)\left[\mathbb{E}_{\Omega^{(0)}}\left(\Psi_{(f)} \Psi_{(k)}\right)-\lambda_{l} \lambda_{k}\right]\left(d \log \lambda_{f}-d \log L_{f}\right),
\end{aligned}
$$

where we use the fact that

$\operatorname{Cov}_{\Omega^{(0)}}\left(\sum_{f \in \mathcal{G}} \Psi_{(f)}\left(d \log \lambda_{f}-d \log L_{f}\right), \frac{\Psi_{(k)}}{\lambda_{k}}\right)=\frac{1}{\lambda_{k}} \sum_{f \in \mathcal{G}}\left[\mathbb{E}_{\Omega^{(0)}}\left(\Psi_{(f)} \Psi_{(k)}\right)-\lambda_{l} \lambda_{k}\right]\left(d \log \lambda_{f}-d \log L_{f}\right)$.

Rearrange this to get

$d \log \lambda_{k}=\frac{1}{\theta} \operatorname{Cov}_{\Omega^{(0)}}\left(d \log \omega_{0}, \frac{\Psi_{(k)}}{\lambda_{k}}\right)+\frac{\theta-1}{\theta} d \log L_{k}-\frac{1}{\lambda_{k}} \frac{(1-\theta)}{\theta} \sum_{f \in \mathcal{G}}\left[\mathbb{E}_{\Omega^{(0)}}\left(\Psi_{(f)} \Psi_{(k)}\right)\right]\left(d \log \lambda_{f}-d \log L_{f}\right)$.

Proof of Proposition 8. Suppose that there is only one capital factor, and conjecture an equilibrium where every labor factor becomes demand-constrained. For each demand- 
constrained factor, from Proposition 7, we have

$$
\begin{aligned}
\frac{1}{\theta} d \log \lambda_{k} & =\frac{1}{\theta} \operatorname{Cov}_{\Omega^{(0)}}\left(d \log \omega_{0}, \frac{\Psi_{(k)}}{\lambda_{k}}\right)+d \log E-\frac{1}{\theta} d \log E \\
& +\frac{1}{\lambda_{k}} \frac{(1-\theta)}{\theta} \mathbb{E}_{\Omega^{(0)}}\left(\Psi_{(\mathcal{D})} \Psi_{(k)}\right) d \log E \\
& -\frac{1}{\lambda_{k}} \frac{(1-\theta)}{\theta} \sum_{f \in \mathcal{S}}\left[\mathbb{E}_{\Omega^{(0)}}\left(\Psi_{(f)} \Psi_{(k)}\right)\right]\left(d \log \lambda_{f}-d \log \bar{L}_{f}\right) \\
\lambda_{k} d \log \lambda_{k} & =\operatorname{Cov}_{\Omega^{(0)}}\left(d \log \omega_{0}, \Psi_{(k)}\right)-(1-\theta) \lambda_{k} d \log E \\
& +(1-\theta) \mathbb{E}_{\Omega^{(0)}}\left(\Psi_{(\mathcal{D})} \Psi_{(k)}\right) d \log E \\
& -(1-\theta) \mathbb{E}_{\Omega^{(0)}}\left(\Psi_{(\mathcal{S})} \Psi_{(k)}\right)\left(d \log \lambda_{\mathcal{S}}-d \log \bar{L}_{\mathcal{S}}\right) .
\end{aligned}
$$

Aggregating over all demand-constrained factors gives

$$
\begin{aligned}
\lambda_{\mathcal{D}} d \log \lambda_{\mathcal{D}}=\sum \sum_{k \in \mathcal{D}} \lambda_{k} d \log \lambda_{k}= & \operatorname{Cov}_{\Omega^{(0)}}\left(d \log \omega_{0}, \Psi_{(\mathcal{D})}\right)-(1-\theta)\left[\lambda_{\mathcal{D}}-\mathbb{E}_{\Omega^{(0)}}\left(\Psi_{(\mathcal{D})}^{2}\right)\right] d \log E \\
& -(1-\theta) \mathbb{E}_{\Omega^{(0)}}\left(\Psi_{(\mathcal{S})} \Psi_{(\mathcal{D})}\right)\left(d \log \lambda_{\mathcal{S}}-d \log \bar{L}_{\mathcal{S}}\right) \\
= & \operatorname{Cov}_{\Omega^{(0)}}\left(d \log \omega_{0}, \Psi_{(\mathcal{D})}\right)-(1-\theta) \mathbb{E}_{\Omega^{(0)}}\left(\Psi_{(\mathcal{D})} \Psi_{(\mathcal{S})}\right) d \log E \\
& +(1-\theta) \mathbb{E}_{\Omega^{(0)}}\left(\Psi_{(\mathcal{S})} \Psi_{(\mathcal{D})}\right) \frac{1}{\lambda_{\mathcal{S}}} \lambda_{\mathcal{D}} d \log \lambda_{\mathcal{D}} \\
& +(1-\theta) \mathbb{E}_{\Omega^{(0)}}\left(\Psi_{(\mathcal{S})} \Psi_{(\mathcal{D})}\right) d \log \bar{L}_{\mathcal{S}} \\
= & \frac{\operatorname{Cov}_{\Omega^{(0)}}\left(d \log \omega_{0}, \Psi_{(\mathcal{D})}\right)}{1-(1-\theta) \frac{1}{\lambda_{\mathcal{S}}} \mathbb{E}_{\Omega^{(0)}}\left(\Psi_{(\mathcal{S})} \Psi_{(\mathcal{D})}\right)} \\
& \frac{-(1-\theta) \mathbb{E}_{\Omega^{(0)}}\left(\Psi_{(\mathcal{D})} \Psi_{(\mathcal{S})}\right) d \log E+(1-\theta) \mathbb{E}_{\Omega^{(0)}}\left(\Psi_{(\mathcal{S})} \Psi_{(\mathcal{D})}\right) d \log \bar{L}_{\mathcal{S}}}{1-(1-\theta) \frac{1}{\lambda_{\mathcal{S}}} \mathbb{E}_{\Omega^{(0)}}\left(\Psi_{(\mathcal{S})} \Psi_{(\mathcal{D})}\right)} .
\end{aligned}
$$


Where we use the fact that $\lambda_{\mathcal{D}} d \log \lambda_{\mathcal{D}}=-\lambda_{\mathcal{S}} d \log \lambda_{\mathcal{S}}$. Finally, combine the equation above with Proposition 2 to get,

$$
\begin{aligned}
d \log Y & =\lambda_{\mathcal{S}} d \log \bar{L}_{\mathcal{S}}+\lambda_{\mathcal{D}} d \log \lambda_{\mathcal{D}}+\lambda_{\mathcal{D}} d \log E \\
& =\frac{\operatorname{Cov}_{\Omega^{(0)}}\left(d \log \omega_{0}, \Psi_{(\mathcal{D})}\right)}{1-(1-\theta) \frac{1}{\lambda_{\mathcal{S}}} \mathbb{E}_{\Omega^{(0)}}\left(\Psi_{(\mathcal{S})} \Psi_{(\mathcal{D})}\right)} \\
& \frac{-(1-\theta) \mathbb{E}_{\Omega^{(0)}}\left(\Psi_{(\mathcal{D})} \Psi_{(\mathcal{S})}\right) d \log E}{1-(1-\theta) \frac{1}{\lambda_{\mathcal{S}}} \mathbb{E}_{\Omega^{(0)}}\left(\Psi_{(\mathcal{S})} \Psi_{(\mathcal{D})}\right)}+\lambda_{\mathcal{D}} d \log E \\
& +\frac{(1-\theta) \mathbb{E}_{\Omega^{(0)}}\left(\Psi_{(\mathcal{S})} \Psi_{(\mathcal{D})}\right) d \log \bar{L}_{\mathcal{S}}}{1-(1-\theta) \frac{1}{\lambda_{\mathcal{S}}} \mathbb{E}_{\Omega^{(0)}}\left(\Psi_{(\mathcal{S})} \Psi_{(\mathcal{D})}\right)}+\lambda_{\mathcal{S}} d \log \bar{L}_{\mathcal{S}} \\
& =\frac{\lambda_{\mathcal{S}} \operatorname{Cov}_{\Omega^{(0)}}\left(d \log \omega_{0}, \Psi_{(\mathcal{D})}\right)}{\lambda_{\mathcal{S}}-(1-\theta) \mathbb{E}_{\Omega^{(0)}}\left(\Psi_{(\mathcal{S})} \Psi_{(\mathcal{D})}\right)} \\
& +\frac{-\lambda_{\mathcal{S}}(1-\theta) \mathbb{E}_{\Omega^{(0)}}\left(\Psi_{(\mathcal{D})} \Psi_{(\mathcal{S})}\right) d \log E}{\lambda_{\mathcal{S}}-(1-\theta) \mathbb{E}_{\Omega^{(0)}}\left(\Psi_{(\mathcal{S})} \Psi_{(\mathcal{D})}\right)}+\lambda_{\mathcal{D}} d \log E \\
& +\lambda_{\mathcal{S}} \frac{(1-\theta) \mathbb{E}_{\Omega^{(0)}}\left(\Psi_{(\mathcal{S})} \Psi_{(\mathcal{D})}\right) d \log \bar{L}_{\mathcal{S}}}{\lambda_{\mathcal{S}}-(1-\theta) \mathbb{E}_{\Omega^{(0)}}\left(\Psi_{(\mathcal{S})} \Psi_{(\mathcal{D})}\right)}+\lambda_{\mathcal{S}} d \log \bar{L}_{\mathcal{S}}
\end{aligned}
$$

Proof of Lemma 2. Define the function $\Phi\left(L^{0}\right) \mapsto L$ by

$$
\begin{aligned}
& w_{f} L_{f}^{0}=\sum_{j \in \mathcal{N}} \Psi_{j f}\left(\frac{w_{f}^{1-\sigma}}{\sum_{k} \Psi_{j k} w_{k}^{1-\sigma}}\right) p_{j} c_{j}, \\
& p_{j} c_{j}=\left(\bar{\Omega}_{0 i}-\kappa_{i}\right) E, \\
& E=\frac{(1-\beta) \sum_{i}\left(1-\kappa_{i}\right)}{\beta} \frac{\bar{E}_{*}}{1+i} \sum_{h} \bar{\lambda}_{h}^{*}\left(\frac{L_{h}^{0}}{L_{h}^{*}}\left(1-\phi_{h}\right)+\phi_{h}\right), \\
& \tilde{w}_{f}=\min \left\{\underline{w}_{f}, w_{f}\right\} \mathbf{1}(f \in \mathcal{L})+w_{f} \mathbf{1}(f \in \mathcal{K}), \\
& L_{f}=\min \left\{\frac{1}{\tilde{w}_{f}} \sum_{j \in \mathcal{N}} \Psi_{j f}\left(\frac{\tilde{w}_{f}^{1-\sigma}}{\sum_{k} \Psi_{j k} \tilde{w}_{k}^{1-\sigma}}\right) p_{j} c_{j}, \bar{L}_{f}\right\} .
\end{aligned}
$$

An equilibrium is when $L^{0}=L$. We show that $\Phi$ is an increasing function mapping $\prod_{f \in \mathcal{G}}\left[0, \bar{L}_{f}\right]$ into itself.

By Lemma $3, w_{-i}$ is increasing and $w_{i}$ is decreasing in $L_{i}^{0}$. This means that $\tilde{w}_{-i}$ is 
increasing in $L_{i}^{0}$ and $\tilde{w}_{i}$ is decreasing in $i$ if $w_{i}>\underline{\mathrm{w}}_{i}$. Hence, $L_{-i}$ is increasing in $L_{i}^{0}$, and $L_{i}$ is increasing in $L_{i}^{0}$. This proves that $\Phi$ is a monotone function, and so we can apply Tarski (1955).

Lemma 3. For the following system of equations

$$
w_{f} L_{f}=\sum_{j \in \mathcal{N}} \Psi_{j f}\left(\frac{w_{f}^{1-\sigma}}{\sum_{k} \Psi_{j k} w_{k}^{1-\sigma}}\right) \Omega_{0 j} E,
$$

$w_{-i}$ is increasing and $w_{i}$ is decreasing in $L_{i}$.

Proof. Start by noting that

$$
\operatorname{Cov}_{\Omega^{(0)}}\left(\Psi_{(f)}, \Psi_{(k)}\right)=\sum_{l} \Omega_{0 l} \Psi_{l f}\left[\Psi_{l k}-\lambda_{k}\right]
$$

Using this fact, and Proposition 10, we can simplify

$$
\begin{aligned}
& \lambda_{k} d \log \lambda_{k}=-(\theta-1) \sum_{f \in \mathcal{G}}\left[-\lambda_{f} \lambda_{k}-\operatorname{Cov}_{\Omega^{(0)}}\left(\Psi_{(f)}, \Psi_{(k)}\right)+1(f=k) \lambda_{k}\right]\left(d \log \lambda_{f}-d \log L_{f}\right) \\
& =\sum_{f \in \mathcal{G}}\left[(1-\theta) \mathbf{1}(f=k) \lambda_{k}-(1-\theta) \lambda_{f} \lambda_{k}-(1-\theta) \operatorname{Cov}_{\Omega^{(0)}}\left(\Psi_{(f)}, \Psi_{(k)}\right)\right]\left(d \log \lambda_{f}-d \log L_{f}\right) \\
& =\sum_{f \in \mathcal{G}}\left[(1-\theta) 1(f=k) \lambda_{k}-(1-\theta) \lambda_{f} \lambda_{k}-(1-\theta) \sum_{l} \Omega_{0 l} \Psi_{l f}\left(\Psi_{k f}-\lambda_{k}\right)\right]\left(d \log \lambda_{f}-d \log L_{f}\right) \\
& =\sum_{f \in \mathcal{G}}\left[(1-\theta) 1(f=k) \lambda_{k}-(1-\theta) \sum_{l} \Omega_{0 l} \Psi_{l f} \Psi_{k f}\right]\left(d \log \lambda_{f}-d \log L_{f}\right) \\
& =\sum_{f \in \mathcal{G}}\left[(1-\theta) \mathbf{1}(f=k) \lambda_{k}-(1-\theta)\left[\mathbb{E}_{\Omega^{(0)}}\left(\Psi_{(f)} \Psi_{(k)}\right)\right]\right]\left(d \log \lambda_{f}-d \log L_{f}\right) \\
& =\sum_{f \in \mathcal{G}}\left[(1-\theta) \mathbf{1}(f=k) \lambda_{k}-(1-\theta)\left[\mathbb{E}_{\Omega^{(0)}}\left(\Psi_{(f)} \Psi_{(k)}\right)\right]\right]\left(d \log \lambda_{f}-d \log L_{f}\right) \\
& =-\sum_{f \in \mathcal{G}}\left[(1-\theta)\left[\mathbb{E}_{\Omega^{(0)}}\left(\Psi_{(f)} \Psi_{(k)}\right)\right]\right]\left(d \log \lambda_{f}-d \log L_{f}\right)+\left[(1-\theta) \lambda_{k}\right]\left(d \log \lambda_{k}-d \log L_{k}\right) \\
& =-(1-\theta) \sum_{f \in \mathcal{G}}\left[\mathbb{E}_{\Omega^{(0)}}\left(\Psi_{(f)} \Psi_{(k)}\right)\right]\left(d \log \lambda_{f}-d \log L_{f}\right)+\left[(1-\theta) \lambda_{k}\right]\left(d \log \lambda_{k}-d \log L_{k}\right)
\end{aligned}
$$

Let

$$
B_{k f}=\left[\mathbb{E}_{\Omega^{(0)}}\left(\Psi_{(f)} \frac{\Psi_{(k)}}{\lambda_{k}}\right)\right]
$$


We know that

$$
\sum_{f} B_{k f}=1
$$

Hence, letting $x=d \log \lambda / d \log L_{i}$ be a column vector and $e_{i}$ the $i$ th basis vector, we can write

$$
\begin{aligned}
\theta x & =-(1-\theta) B x-(I-(1-\theta) B) e_{i} \\
x & =-(\theta I+(1-\theta) B)^{-1}(I-(1-\theta) B) e_{i}=-A(I-(1-\theta) B) e_{i} .
\end{aligned}
$$

By Lemma 4, $A=(\theta I+(1-\theta) B)^{-1}$ is an M-matrix, hence by $A_{5}$ of Theorem 6.2 .3 of Berman and Plemmons (1979), $-A(I-(1-\theta) B) e_{i}$ has the same signs as $-(I-(1-\theta) B)$. Since $-(I-(1-\theta) B)$ has negative diagonal and positive off-diagonal elements, this means that $x_{i}$ is negative and $x_{-i}$ is positive, as needed.

Lemma 4. The matrix defined in Lemma 4

$$
A=(\theta I+(1-\theta) B)^{-1}
$$

is an M-matrix.

Proof. By Theorem 6.2.3 of Berman and Plemmons (1979), it suffices to prove that $A^{-1}$ has all positive elements and that $A$ is a Z-matrix. The fact that $A^{-1}$ has all positive elements is immediate from its definition. To show that $A$ is a Z-matrix, note that we can write

$$
\begin{aligned}
A & =(\theta I+(1-\theta) B)^{-1}, \\
& =(I-(1-\theta)(I-B))^{-1}, \\
& =\sum_{n=0}^{\infty}(1-\theta)^{n}(I-B)^{n} .
\end{aligned}
$$

Hence, since $I-B$ is an $M$-matrix, $(1-\theta)(I-B) X$ does not change the sign of the columns of $X$ for any $X$. Hence, by induction, and the fact that $M$-matrices are closed under addition, we have that $A$ has the same sign as the elements of $(I-B)$, and hence $A$ is a Z-matrix.

Proof of Proposition 9. To prove the statements regarding $\Delta \log L$, we use Theorem 3 from Milgrom and Roberts (1994). Since $\Delta \log Y$ is a monotone function of $\Delta \log L$, this also establishes the results about real GDP. It remains to prove the claims regarding inflation. To prove that labor supply shocks (on their own) are inflationary, we need to show that 
the price level $p^{Y}$ is decreasing in by $\bar{L}$. To do so, consider some negative labor shocks then

$$
\begin{aligned}
\Delta \log p^{Y} & =\Delta \log E-\Delta \log Y \\
& \geq \Delta \log E-\sum_{f} \bar{\lambda}_{f} \Delta \log L_{f} \\
& =\Delta \log \left(\sum_{h} \bar{\lambda}_{h}^{*}\left(\frac{L_{h}}{L_{h}^{*}}\left(1-\phi_{h}\right)+\phi_{h}\right)\right)-\sum_{f} \bar{\lambda}_{f} \Delta \log L_{f} \\
& \geq \Delta \log \left(\sum_{h} \bar{\lambda}_{h}^{*} \frac{L_{h}}{L_{h}^{*}}\right)-\sum_{f} \bar{\lambda}_{f} \Delta \log L_{f} \\
& =\Delta \log \left(\sum_{h} \bar{\lambda}_{h}^{*} \exp \left(\log L_{h} / L_{h}^{*}\right)\right)-\sum_{f} \bar{\lambda}_{f} \Delta \log L_{f} \\
& \geq \sum_{h} \bar{\lambda}_{h}^{*} \Delta \log L_{h}-\sum_{f} \bar{\lambda}_{f} \Delta \log L_{f}=0,
\end{aligned}
$$

as long as $\bar{\lambda}^{*}=\bar{\lambda}$. The second line follows from the fact that $\log Y$ is log-concave (see Baqaee and Farhi, 2019b).

To prove that aggregate demand shocks, like forward guidance shocks, (on their own) are deflationary, we need to show that the price level $p^{Y}$ is increasing $E_{*} /(1+i)$. To do so, consider some shock then

$$
\begin{aligned}
\Delta \log p^{Y} & =\Delta \log E-\Delta \log Y \\
& \geq \Delta \log E-\sum_{f} \bar{\lambda}_{f} \Delta \log L_{f} \\
& =\Delta \log \left(\frac{(1-\beta) \sum_{i}\left(1-\kappa_{i}\right)}{\beta} \frac{\bar{E}_{*}}{1+i} \sum_{h} \bar{\lambda}_{h}^{*}\left(\frac{L_{h}}{L_{h}^{*}}\left(1-\phi_{h}\right)+\phi_{h}\right)\right)-\sum_{f} \bar{\lambda}_{f} \Delta \log L_{f} \\
& \geq \Delta \log \left(\frac{\bar{E}_{*}}{1+i}\right)-\sum_{f} \bar{\lambda}_{f} \Delta \log L_{f} \\
& \geq \Delta \log \left(\frac{\bar{E}_{*}}{1+i}\right)-\sum_{f} \bar{\lambda}_{f} \Delta \log \left(\frac{\bar{E}_{*}}{1+i}\right) \\
& \geq 0 .
\end{aligned}
$$

Proof of Proposition 10. This follows from an application of Proposition 9 in Baqaee and Farhi (2019a). 


\section{Appendix C A Microfoundation for Demand Shocks}

When household preferences are Cobb-Douglas, there is a simple microfoundation for the demand shocks motivated by health concerns. To see this, consider households with log preferences

$$
(1-\beta)\left[\sum_{i \in \mathcal{N}} \bar{\Omega}_{0 i} \log c_{i}-H\left(\left\{c_{i}\right\}_{i \in \mathcal{N}}\right)\right]+\beta \sum_{i} \bar{\Omega}_{0 i} \log c_{i}^{*},
$$

where $\beta \in[0,1]$ captures households' time-preferences, and $c_{i}$ and $c_{i}^{*}$ are current and future consumption of good $i$. The function $H\left(\left\{c_{i}\right\}_{i \in \mathcal{N}}\right)$ is a homothetic aggregator that captures health concerns of the household associated with consumption today. We assume there are no health concerns in the future. We let the disutility of consumption due to health concerns be

$$
H\left(\left\{c_{i}\right\}_{i \in \mathcal{N}}\right)=\sum_{i} \kappa_{i} \log c_{i}
$$

where $\kappa_{i} \geq 0$ captures the riskiness of consuming good $i$. As $\kappa_{i}$ increases, households choose to spend a smaller fraction of their permanent income on purchasing $i$. We call an increase in $\kappa_{i}$ an individual negative demand shock for sector $i$ (in contrast to aggregate demand shocks which affect spending on all goods produced this period).

The health-risk parameters $\kappa$ then map into shocks to both the intersectoral composition of demand

$$
\Delta \log \omega_{0 i}=\Delta \log \frac{\bar{\Omega}_{0 i}-\kappa_{i}}{\left(1-\sum_{j \in \mathcal{N}} \kappa_{j}\right) \bar{\Omega}_{0 i}},
$$

and shocks to aggregate demand

$$
\Delta \log \zeta=-\Delta \log (1+i)-\Delta \log \frac{\beta}{1-\beta}+\Delta \log \bar{E}_{*}+\Delta \log \left(1-\sum_{j \in \mathcal{N}} \kappa_{j}\right)
$$

For future reference, when we refer to an aggregate demand shock, we mean a change in $\Delta \log \zeta$ that keeps the intersectoral composition of final demand $\Delta \log \omega_{0}=0$ constant.

\section{Appendix D Extension: Generalizing to Arbitrary CES Economies}

This appendix shows how Proposition 7 generalizes to arbitrary nested-CES production networks. ${ }^{28}$ To do this, suppose that each good $i \in \mathcal{N}$ is produced with the production

\footnotetext{
${ }^{28}$ Our results can easily be extended beyond the nested-CES case along the lines of Section 5 in Baqaee and Farhi (2019a).
} 
function

$$
\frac{y_{i}}{\bar{y}_{i}}=\frac{A_{i}}{\bar{A}_{i}}\left(\sum_{j \in \mathcal{N}+\mathcal{G}} \bar{\omega}_{i j}\left(\frac{x_{i j}}{\bar{x}_{i j}}\right)^{\frac{\theta_{i}-1}{\theta_{i}}}\right)^{\frac{\theta_{i}}{\theta_{i}-1}}
$$

where we now allow the elasticity of substitution $\theta_{i}$ to vary across producers. The households' consumption function is

$$
\frac{y_{0}}{\bar{y}_{0}}=\left(\sum_{j \in \mathcal{N}+\mathcal{G}} \bar{\omega}_{0 j} \frac{\omega_{0 j}}{\bar{\omega}_{0 j}}\left(\frac{x_{0 j}}{\bar{x}_{0 j}}\right)^{\frac{\theta_{0}-1}{\theta_{0}}}\right)^{\frac{\theta_{0}}{\theta_{0}-1}}
$$

where $\omega_{0 j}$ are sectoral demand shocks with $\sum_{j} \omega_{0 j}=1$. where $x_{i j}$ are intermediate inputs from $j$ used by $i$. In these equations, variables with over-lines are normalizing constants.To simplify the notation below, we think of $\omega_{0}$ as a $1 \times(1+\mathcal{N}+\mathcal{G})$ vector with $k$-th element $\omega_{0 k}$.

We now show how changes in factor income shares $d \log \lambda_{f}$ are determined, which along with Propositions 1 and 2 pins down output, employment, and inflation. Recall that for a matrix $M$, we denote by $M_{(i)}$ its $i$-th row by $M^{(j)}$ its $j$-th column. We write $\operatorname{Cov}_{\Omega^{(j)}}(\cdot, \cdot)$ to denote the covariance of two vectors of size $1+\mathcal{N}+\mathcal{G}$ using the $j$-the row of the input-ouput matrix $\Omega^{(j)}$ as a probability distribution.

Proposition 10 (Propagation). Changes in sales and factor shares are given by

$$
\begin{aligned}
d \log \lambda_{k} & =\theta_{0} \operatorname{Cov}_{\Omega^{(0)}}\left(d \log \omega_{(0)}, \frac{\Psi_{(k)}}{\lambda_{k}}\right) \\
& +\sum_{j \in 1+\mathcal{N}} \lambda_{j}\left(\theta_{j}-1\right) \operatorname{Cov}_{\Omega^{(j)}}\left(\sum_{i \in \mathcal{N}} \Psi_{(i)} d \log A_{i}-\sum_{f \in \mathcal{G}} \Psi_{(f)}\left(d \log \lambda_{f}-d \log L_{f}\right), \frac{\Psi_{(k)}}{\lambda_{k}}\right)
\end{aligned}
$$

almost everywhere, where changes in factor employments are given by

$$
d \log L_{f}=\left\{\begin{array}{cc}
d \log \bar{L}_{f}, & \text { for } \quad f \in \mathcal{K}, \\
\min \left\{d \log \lambda_{f}+d \log E, d \log \bar{L}_{f}\right\}, & \text { for } f \in \mathcal{L} .
\end{array}\right.
$$

The intuition for Proposition 10 is similar to that of Proposition 7. Changes in factor shares depend on changes in the composition of final demand and on relative prices:

$$
d \log \lambda_{k}=\theta_{0} \operatorname{Cov}_{\Omega^{(0)}}\left(d \log \omega_{0}, \Psi_{(k)} / \lambda_{k}\right)+\sum_{j \in 1+\mathcal{N}} \lambda_{j}\left(\theta_{j}-1\right) \operatorname{Cov}_{\Omega^{(j)}}\left(-d \log p, \Psi_{(k)} / \lambda_{k}\right) .
$$


The first term on the right-hand side $\theta_{0} \operatorname{Cov}_{\Omega^{(0)}}\left(d \log \omega_{0}, \Psi_{(k)} / \lambda_{k}\right)$ on the right-hand side is the direct effect of shocks to the sectoral composition of final demand on the sales of $k$.The second term $\sum_{j \in 1+\mathcal{N}} \lambda_{j}\left(\theta_{j}-1\right) \operatorname{Cov}_{\Omega^{(j)}}\left(-d \log p, \Psi_{(k)} / \lambda_{k}\right)$ on the right-hand side captures the changes in the sales of $k$ from substitutions by producers $j$ downstream from $k$. If producer $j$ has an elasticity of substitution $\theta_{j}$ below one so that its inputs are complements, then it shifts its expenditure towards those inputs with higher price increases, and this increases the demand for $k$ if those inputs also use $k$ intensively (measured by $\Psi_{l k} / \lambda_{k}$ ). The result follows from noticing that changes in relative prices are, in turn, given by changes in factor shares

$$
d \log p_{k}-d \log E=-\sum_{i \in \mathcal{N}} \Psi_{k i} d \log A_{i}+\sum_{f \in \mathcal{G}} \Psi_{k f} d \log \left(d \log \lambda_{f}-d \log L_{f}\right),
$$

where we use the fact that $d \log w_{f}-d \log E=d \log \lambda_{f}+d \log E-d \log L_{f}$.

\section{Appendix E Extension: Semi-Flexible Wages}

In practice, we might imagine that wages can fall albeit not by enough to clear the market. For each factor $f \in \mathcal{L}$, suppose the following conditions hold

$$
\left(\frac{w_{f}}{\bar{w}_{f}}-\left(\frac{L_{f}}{\bar{L}_{f}}\right)^{\frac{1}{\gamma_{f}}}\right)\left(L-\bar{L}_{f}\right)=0, \quad L_{f} \leq \bar{L}_{f}, \quad\left(\frac{L_{f}}{\bar{L}_{f}}\right)^{\frac{1}{\gamma_{f}}} \leq \frac{w_{f}}{\bar{w}_{f}} .
$$

The parameter $\gamma_{f}$ controls the degree of downward wage flexibility. If $\gamma_{f}=\infty$, then the wage is perfectly rigid downwards. If $\gamma_{f}=0$, then the wage is fully flexible, and we recover the neoclassical case.

\section{E.1 Generalizing the Results}

Collectively, Propositions 1 and 2, as well as Proposition 10 in Appendix D, pin down all equilibrium outcomes. So, we discuss how each can be generalized. Proposition 1 remains exactly the same as before, so we do not restate it. The only change to Proposition 
2 is that we now have

$$
\begin{aligned}
d \log Y & =\sum_{i \in \mathcal{N}} \lambda_{i} d \log A_{i}+\sum_{f \in \mathcal{G}} \lambda_{f} d \log L_{f}, \\
& =\sum_{i \in \mathcal{N}} \lambda_{i} d \log A_{i}+\sum_{f \in \mathcal{G}} \lambda_{f} d \log \bar{L}_{f}+\sum_{f \in \mathcal{L}} \frac{\gamma_{f}}{1+\gamma_{f}} \lambda_{f} \min \left\{d \log \lambda_{f}+d \log E-d \log \bar{L}_{f}, 0\right\},
\end{aligned}
$$

In particular, this implies that Corollary 1 about the behavior of inflation remains unchanged:

$$
d \log p^{Y}=\frac{1}{\rho} d \log \zeta-\frac{1}{\rho} \sum_{i \in \mathcal{N}} \lambda_{i} d \log A_{i}-\frac{1}{\rho} \sum_{f \in \mathcal{G}} \phi_{f} \lambda_{f} d \log L_{f} .
$$

Hence, reductions in employment are still inflationary in the absence of exogenous negative demand shocks.

The only endogenous objects left to be determined are the factor shares. Proposition 10 can be generalized to pin down factor shares. In particular, changes in factor shares solve the following linear system:

$$
\begin{aligned}
d \log \lambda_{k} & =\theta_{0} \operatorname{Cov}_{\Omega^{(0)}}\left(d \log \omega_{(0)}, \frac{\Psi_{(k)}}{\lambda_{k}}\right)+\sum_{j \in 1+\mathcal{N}} \lambda_{j}\left(\theta_{j}-1\right) \operatorname{Cov}_{\Omega^{(j)}}\left(\sum_{i \in \mathcal{N}} \Psi_{(i)} d \log A_{i}\right. \\
& \left.-\sum_{f \in \mathcal{G}} \Psi_{(f)}\left(d \log \lambda_{f}-d \log L_{f}+d \log E\right), \frac{\Psi_{(k)}}{\lambda_{k}}\right)
\end{aligned}
$$

where

$$
d \log L_{f}=\left\{\begin{array}{cc}
\frac{\gamma_{f}}{1+\gamma_{f}}\left(d \log \lambda_{f}+d \log E\right)+\frac{1}{1+\gamma_{f}} d \log \bar{L}_{f} & \text { if } f \in \mathcal{D} \\
d \log \bar{L}_{f} & \text { if } f \in \mathcal{S} .
\end{array}\right.
$$

\section{Appendix F Extension: Investment}

To model investment, we add intertemporal production functions into the model. An investment function transforms goods and factors in the present period into goods that can be used in the future. In this case, instead of breaking the problem into an intertemporal and intratemporal problem, we must treat both problems at once. In this section, we first discuss the general local comparative statics with investment, extending the results in Section 3, then we discuss a special case with simple sufficient statistics and global comparative statics, extending the results in Section 5.2.

In the body of the paper, we assumed that prices in the future $p_{*}^{Y}$ were fixed, which 
meant that nominal expenditures in the future were also fixed $p_{*}^{Y} Y_{*}=E_{*}$. In the version of the model with investment, output in the future $Y_{*}$ is not exogenous, so assuming $p_{*}^{Y}$ is no longer equivalent to assuming $E_{*}$ is fixed. Therefore, we consider both situations.

\section{F.1 General local comparative statics}

When we add investment to the model, we can still use Proposition 2 without change. However, we can no longer use the Euler equation to pin down nominal expenditures today, since nominal GDP today includes investment expenditures and output tomorrow can no longer taken to be exogenous. Instead, to determine $d \log E$, we must use a version of Proposition 10. For this subsection, we assume that nominal expenditures in the future period are fixed and we denote the future period by *

In particular, let $\lambda_{i}^{I}$ denote the intertemporal sales share - expenditures on quantity $i$ as a share of the net present value of household income. Furthermore, let $\bar{\Omega}^{I}$ represent the intertemporal input-output matrix, which includes the capital accumulation equations. Then, letting intertemporal consumption be the zero-th good, and abstracting from shocks to the sectoral composition of demand for simplicity, we can write

$$
d \log \lambda_{k}^{I}=\sum_{j} \lambda_{j}^{I}\left(\theta_{j}-1\right) \operatorname{Cov}_{\Omega^{I,(j)}}\left(\sum_{i \in \mathcal{N}} \Psi_{(i)}^{I} d \log A_{i}-\sum_{f \in \mathcal{G}} \Psi_{(f)}^{I}\left(d \log \lambda_{f}^{I}-d \log L_{f}\right), \frac{\Psi_{(k)}^{I}}{\lambda_{k}^{I}}\right)
$$

almost everywhere, where changes in factor employments are given by

$$
d \log L_{f}=\left\{\begin{array}{cc}
d \log \bar{L}_{f}, & \text { for } f \in \mathcal{K}, \\
\min \left\{d \log \lambda_{f}^{I}-d \log \lambda_{*}^{I} d \log \bar{L}_{f}\right\}, & \text { for } f \in \mathcal{L} .
\end{array}\right.
$$

This follows from the fact that nominal expenditures on each factor $f$ is given by $d \log \lambda_{f}^{I}+$ $d \log E^{I}$, where $E^{I}$ is the net-present value of household income. However, since nominal expenditures in the future are fixed, we have $d \log E_{*}=d \log \lambda_{*}^{I}+d \log E^{I}=0$. This allows us to write nominal expenditures on each factor as $d \log \lambda_{f}^{I}-d \log \lambda_{*}^{I}$.

\section{F.2 Global Comparative Statics}

We can extend the results in Section 5.2 to the model with investment. To do so, we assume that the intertemporal elasticity of substitution $\rho$ is the same as the intersectoral elasticities of substitution $\rho=\theta_{j}=\theta$ for every $j \in \mathcal{N}$. In this case, the initial factor shares are, once again, a sufficient statistic for the production network. Furthermore, we can also 
prove that the set of equilibria form a lattice under some additional assumptions.

Proposition 11. Suppose that the intertemporal elasticity of substitution, the elasticities of substitution in production and in final demand are all the same $\theta$. Suppose that there are only shocks to potential factor supplies $\Delta \log \bar{L}$. If future nominal expenditure is fixed, then assuming in addition that $\theta<1$, there is a unique best and worst equilibrium: for any other equilibrium, $\Delta \log L$ are lower than at the best and higher than at the worst. Furthermore, both in the best and in the worst equilibrium, $\Delta \log$ L are increasing in $\Delta \log \bar{L}$.

Intuitively, a negative shock to potential factor supply today potentially reduces output tomorrow by reducing resources available for consumption tomorrow. Since nominal expenditures tomorrow are fixed, this raises the price level tomorrow. If the elasticity of substitution $\theta$ is less than one, then the increase in the price level tomorrow reduces expenditures on non-shocked factor markets and potentially causes them to become slack.

In Proposition 11, we assume that nominal expenditures in the final period are fixed. If instead we assume that the nominal price level in the future is fixed, rather than nominal expenditures, then Proposition 11 applies regardless of the value of the elasticity of substitution $\theta$.

\section{Appendix G Extension: Bankruptcies}

The paper abstracts from capital market frictions and bankruptcies. In this appendix, we briefly discuss how our results can be extended to the case with these frictions. We begin by generalizing our comparative statics to a case with firm exits. We then make three observations: (1) in a production network, the negative effects of demand shocks are amplified if there are exits because of an intermediate-input multiplier; (2) exits, by acting as endogenous negative supply shocks, can change the flow of spending and cause Keynesian spillovers outside of the Cobb-Douglas case; and, (3) firm failures, by potentially destroying intangible firm-specific capital, can reduce output in the future, and by lowering output in the future, reduce aggregate demand today through the Euler equation.

\section{G.1 Local Comparative Statics with Bankruptcies}

To capture firm failures, we modify the general structure described in Section 2 as follows. We assume that output in sector $i \in \mathcal{N}$ is a CES aggregate of identical producers $j$ each with constant returns production functions $y_{i k}=A_{i} f_{i}\left(x_{i j}^{k}\right)$, where $x_{i j}^{k}$ is the quantity of industry 
$j$ 's output used by producer $k$ in industry $i$. Assuming all firms within an industry use the same mix of inputs, sectoral output is

$$
y_{i}=\left(\int y_{i k}^{\frac{\sigma_{i}-1}{\sigma_{i}}} d k\right)^{\frac{\sigma_{i}}{\sigma_{i}-1}}=M_{i}^{\frac{1}{\sigma_{i}-1}} A_{i} f_{i}\left(x_{i j}\right),
$$

where $x_{i j}$ is the quantity of input $j$ used by industry $i, M_{i}$ is the mass of producers in industry $i, \sigma_{i}>1$ is the elasticity of substitution across producers, and $A_{i}$ is an exogenous productivity shifter. From this equation, we see that a change in the mass of operating firms acts like a productivity shock and changes the industry-level price. Therefore, if shocks outside sector $i$ trigger a wave of exits, then this will set in motion endogenous negative productivity shock $\left(1 /\left(\sigma_{i}-1\right)\right) \Delta \log M_{i}$ in sector $i$.

Suppose that each firm must maintain a minimum level of revenue in order to continue operation. $.^{29}, 30$ We are focused on a short-run application, so we do not allow new entry, but of course, this would be important for long-run analyses. ${ }^{31}$

The mass of firms that operate in equilibrium is therefore given by

$$
M_{i}=\min \left\{\frac{\lambda_{i} E}{\bar{\lambda}_{i} \bar{E}} \bar{M}_{i}, \bar{M}_{i}\right\}
$$

where $\bar{M}_{i}$ is the exogenous initial mass of varieties, $\lambda_{i} E$ is nominal revenue earned by sector $i$ and $\bar{\lambda}_{i} \bar{E}$ is the initial nominal revenue earned by $i$. If nominal revenues fall relative to the baseline, then the mass of producers declines to ensure that sales per producer remain constant. In order to capture government-mandated shutdowns of certain firms, we allow for shocks that reduce the exogenous initial mass of producers $\Delta \log \bar{M}_{i} \leq 0$.

We can generalize Propositions 2 and 10 to this context. The only difference is that we must replace $d \log A_{i}$ by $d \log A_{i}+\left(1 /\left(\sigma_{i}-1\right)\right) d \log M_{i}$, where

$$
d \log M_{i}=d \log \bar{M}_{i}+\min \left\{d \log \lambda_{i}+d \log E-d \log \bar{M}_{i}, 0\right\} .
$$

\footnotetext{
${ }^{29}$ One possible micro-foundation is each producer must pay its inputs in advance by securing withinperiod loans and that these loans have indivisibilities: only loans of size greater than some minimum level can be secured. This minimum size is assumed to coincide with the initial costs $\bar{\lambda}_{i} \bar{E} / \bar{M}_{i}$ of the producer.

${ }^{30}$ Another possible micro-foundation is as follows. Producers within a sector charge a CES markup $\mu_{i}=\sigma_{i} /\left(\sigma_{i}-1\right)$ over marginal cost. These markups are assumed to be offset by corresponding production subsidies. Producers have present nominal debt obligations corresponding to their initial profits $(1-$ $\left.1 / \mu_{i}\right) \bar{\lambda}_{i} \bar{E} / \bar{M}_{i}$. The same is true in the future. If present profits $\left(1-1 / \mu_{i}\right) \lambda_{i} E / M_{i}$ fall short of the required nominal debt payment $\left(1-1 / \mu_{i}\right) \bar{\lambda}_{i} \bar{E} / \bar{M}_{i}$, then the firm goes bankrupt and exits. Alternatively, we can imagine that there is no future debt obligation but that firms cannot borrow.

${ }^{31}$ See Baqaee (2018) and Baqaee and Farhi (2020a) for production networks with both entry and exit.
} 
This backs up the claim that the $d \log M_{i}$ 's act like endogenous negative productivity shocks. They provide a mechanism whereby a negative demand shock, say in the composition of demand or in aggregate demand $d \log \zeta$, triggers exits that are isomorphic to negative supply shocks.

As in the other examples, the general lesson is that the output response, to a firstorder, is again given by an application of Hulten's theorem along with an amplification effect which depends on how the network redistributes demand and triggers Keynesian unemployment in some factors and firm failures in some sectors.

Having generalized the local comparative statics, we now make three observations about the way bankruptcies can propagate and affect aggregates. In order to simplify the exposition, we abstract away from HtM households for the rest of the section.

\section{G.2 Intermediate Multiplier of Bankruptcies}

If there are increasing returns, then firm failures can also affect supply today directly. As the economy scales down, marginal cost goes up. Our formulation of industry-level production functions (G.1) have this property due to the love-of-variety effect. Hence, firm exits act like negative TFP shocks, and if there are intermediate inputs, then these endogenous negative TFP shocks are amplified.

To see this, consider a Cobb-Douglas model where $\rho=\theta_{0}=\theta_{j}=1$ and negative demand shocks. In this case, since there are no HtM households, the effect on output is given by

$$
d \log Y=\sum_{i \in \mathcal{N}} \lambda_{i} \frac{1}{\sigma_{i}-1} d \log M_{i}=\sum_{i \in \mathcal{N}} \lambda_{i} \frac{1}{\sigma_{i}-1}\left(d \log \lambda_{i}+d \log E\right) .
$$

Using the fact that $d \log \lambda_{i}+d \log E=-\sum_{j \in \mathcal{N}} \Psi_{j i} d \kappa_{j} / \lambda_{i}$, we can write

$$
d \log Y=-\sum_{i \in \mathcal{N}} \frac{1}{\sigma_{i}-1} \sum_{j \in \mathcal{N}} \Psi_{j i} d \kappa_{j}
$$

Hence, the higher is the use of intermediate inputs, the larger are the elements of the Leontief inverse $\Psi$, and the larger is the negative effect on output. Intuitively, a reduction in demand causes exits at every step in the supply chain, and so the longer the supply chains, the more costly the exits. 


\section{G.3 Bankruptcies and Expenditure Switching}

In the previous example, we deliberately chose a Cobb-Douglas economy since the expenditure shares do not respond to relative prices. If the elasticities of substitution are not all equal to one, the endogenous TFP shocks associated with exits, by changing expenditure shares and the flow of spending, can trigger additional cascades of unemployment and failure.

To make this concrete, consider a simple example economy without intermediate inputs where each sector uses only its own labor. Assume that there are no shocks to aggregate demand $(d \log \zeta=0)$. Set the intertemporal elasticity of substitution $\rho=1$ and share of HtM households $1-\phi=0$ to ensure that nominal expenditure is constant $(d \log E=0)$. We also assume that there are no exogenous shocks to productivities $\left(d \log A_{i}=0\right)$, no shocks to potential labor $\left(d \log \bar{L}_{f}=0\right)$, and no shocks to the sectoral composition of demand $\left(d \log \omega_{0 j}=0\right)$. Finally, we assume that all sectors have the same within-sector elasticity of substitution $\sigma_{i}=\sigma>1$.

We focus on exogenous shocks $d \log \bar{M}_{i} \leq 0$ capturing government-mandated shutdowns. We show how endogenous failures can amplify these negative supply shocks. The insights are more general and also apply to shocks to potential labor. Similarly, failures can be triggered by negative aggregate demand shocks, and the resulting endogenous negative supply shocks can result in stagflation with simultaneous reductions in output and increases in inflation.

We start by analyzing the case where sectors are complements, and then consider the case where they are substitutes. For brevity, we jump directly to the final result and leave the derivations in a different appendix - Appendix $\mathrm{H}$.

Shut-down shock with complements. Assume that sectors are complements $(\theta<1)$ and consider the government-mandated shutdown of some firms in only one sector $i$. The change in output is given by

$$
d \log Y=\lambda_{i} \frac{1}{\sigma-1} d \log \bar{M}_{i}+\frac{(1-\theta)\left(1-\lambda_{i}\right) \frac{\sigma}{\sigma-1}}{1-(1-\theta)\left(1-\lambda_{i}\right)\left(1-\frac{1}{\sigma-1} \frac{\lambda_{i}}{1-\lambda_{i}}\right)} \lambda_{i} \frac{1}{\sigma-1} d \log \bar{M}_{i} .
$$

The first term on the right-hand side is the direct reduction in output from the shut-down in sector $i$. The second term capture the further indirect equilibrium reduction in output due to firm failures and Keynesian unemployment in the other sectors. Intuitively, the shutdown in sector $i$ raises the relative price of $i$, and because of complementarities, demand in the rest of the sectors falls. This reduction in nominal spending causes unemployment 
and additional exits in the other sectors.

Shut-down shock with substitutes. Consider the same experiment as above but assume now that sectors are substitutes $(\theta>1)$. Shut-downs in $i$ raise the price of $i$ relative to other sectors, and cause substitution away from $i$. As long as the elasticity of substitution within the sector $\sigma>1$ is large enough and the elasticity of substitution across sectors $\theta>1$ is not too large, the shut-down in sector $i$ causes unemployment in sector $i$, but no additional firm failures in sector $i$. Furthermore, the other sectors maintain full employment and experiences no failures. In this case the response of output is given by

$$
d \log Y=\lambda_{i} \frac{1}{\sigma-1} d \log \bar{M}_{i}+\frac{\left(\theta_{0}-1\right)\left(1-\lambda_{i}\right)}{1-\left(\theta_{0}-1\right) \lambda_{i}} \lambda_{i} \frac{1}{\sigma-1} d \log \bar{M}_{i}
$$

where the first term on the right-hand side is the direct effect of the shutdown and the second term is the amplification from the indirect effect of the shutdown which results in Keynesian unemployment in $i$.

\section{G.4 Scarring Effect of Bankruptcies}

One of the primary concerns about firm failures is that it results in the destruction of irreversible investments. This lowers output in the future, and through the Euler equation, depresses spending today. ${ }^{32}$ In other words, the destruction of irreversible investments can act like an endogenous negative aggregate demand shock. To see this, for simplicity, assume there are no HtM agents and suppose that when firms exit in the first period $d \log M$, they do not return in the next period.

In particular, by the Envelope theorem, output in the future falls by

$$
d \log Y_{*}=\sum_{i \in \mathcal{N}} \frac{\lambda_{i}^{*}}{\sigma_{i}-1} d \log M_{i}=\sum_{i \in \mathcal{S}} \frac{\lambda_{i}^{*}}{\sigma_{i}-1} d \log \bar{M}_{i}+\sum_{i \in \mathcal{D}} \frac{\lambda_{i}^{*}}{\sigma_{i}-1}\left(d \log \lambda_{i}+d \log E\right) .
$$

The endogenous changes in $d \log Y_{*}$ then mean that the previously exogenous aggregate demand shock $d \log \zeta$, defined by (3.3) now contains an endogenous term

$$
d \log \zeta=-\rho\left(d \log (1+i)+\frac{1}{1-\beta} d \log \beta-d \log \bar{p}_{*}^{Y}\right)+d \log Y_{*} .
$$

However, the rest of the model remains the same. We can combine the Euler equation in

\footnotetext{
${ }^{32}$ This mechanism is the same as the one emphasized by Benigno and Fornaro (2018), except here it corresponds to the destruction of irreversible investment instead of reduced investment in innovation.
} 
(3.4), with the aggregation and propagation equations in Propositions 2 and 10 (remembering that $d \log A_{i}$ should be replaced by $\left.d \log A_{i}+d \log M_{i} /\left(\sigma_{i}-1\right)\right)$.

\section{Appendix H Detailed Derivations for Example with Fail- ures}

Preliminaries. Changes in the sales of $i$ are given by

$$
d \log \lambda_{i}=\left(1-\theta_{0}\right)\left(1-\lambda_{i}\right)\left(d \log p_{i}-\sum_{j \in \mathcal{N}} \lambda_{j} d \log p_{j}\right)
$$

where changes in the price of $i$ depend on changes in the wage in $i$ and on the endogenous reduction in the productivity of $i$ driven by firm failures

$$
d \log p_{i}=d \log w_{i}-\frac{1}{\sigma-1} d \log M_{i}
$$

The change in wages in $i$ are given by

$$
d \log w_{i}=\max \left\{d \log \lambda_{i}-d \log \bar{L}_{i}, 0\right\},
$$

and changes in the mass of producers in $i$ are given by

$$
d \log M_{i}=\min \left\{d \log \lambda_{i}, d \log \bar{M}_{i}\right\}
$$

We consider the effect of shutdown shocks $d \log \bar{M}_{i}$ starting with the case where sectors are complements and then the case where they are substitutes. The effect of negative labor shocks $d \log \bar{L}_{i}$ is similar.

Shut-down shock with complements. Assume that sectors are complements $(\theta<1)$ and consider the government-mandated shutdown of some firms in only one sector $i$. We can aggregate the non-shocked sectors into a single representative sector indexed by $-i$. We therefore have $d \log \bar{M}_{i}<0=d \log \bar{M}_{-i}$.

The closures of firms in $i$ raise its price $\left(d \log p_{i}>0\right)$, which because of complementarities, increases its share $\left(d \log \lambda_{i}>0\right)$. It therefore does not trigger any further endogenous exit in this shocked sector $\left(d \log M_{i}=d \log \bar{M}_{i}\right)$. In addition, the wages of its workers increases $\left(d \log w_{i}>0\right)$. The shock reduces expenditure on the other sectors $\left(d \log \lambda_{-i}<0\right)$, and this reduction in demand triggers endogenous exits $\left(d \log M_{-i}<0\right)$, pushes wages 
against their downward rigidity constraint $\left(d \log w_{-i}=0\right)$ and creates unemployment $\left(d \log L_{-i}<0\right)$, both of which endogenously amplify the reduction in output through failures and Keynesian effects.

Using equations (H.1), (H.2), (H.3), and (H.4), we find

$$
\begin{gathered}
d \log \lambda_{i}=-\frac{(1-\theta)\left(1-\lambda_{i}\right)}{1-(1-\theta)\left(1-\lambda_{i}\right)\left(1-\frac{1}{\sigma-1} \frac{\lambda_{i}}{1-\lambda_{i}}\right)} \frac{1}{\sigma-1} d \log \bar{M}_{i}>0, \\
d \log M_{-i}=d \log L_{-i}=-\frac{\lambda_{i}}{1-\lambda_{i}} d \log \lambda_{i}<0,
\end{gathered}
$$

and finally

$$
d \log Y=\lambda_{i} \frac{1}{\sigma-1} d \log \bar{M}_{i}+\frac{(1-\theta)\left(1-\lambda_{i}\right) \frac{\sigma}{\sigma-1}}{1-(1-\theta)\left(1-\lambda_{i}\right)\left(1-\frac{1}{\sigma-1} \frac{\lambda_{i}}{1-\lambda_{i}}\right)} \lambda_{i} \frac{1}{\sigma-1} d \log \bar{M}_{i} .
$$

The first term on the right-hand side is the direct reduction in output from the shut-down in sector $i$. The second term capture the further indirect equilibrium reduction in output via firm failures and Keynesian unemployment in the other sectors.

Shut-down shock with substitutes. Consider the same experiment as above but assume now that sectors are substitutes $(\theta>1)$. We conjecture an equilibrium where sales in sector $i$ do not fall more quickly than the initial shock $d \log \lambda_{i}-d \log \bar{M}_{i}>0$. Sector $i$ loses demand following the exogenous shutdown of some of its firms, and this results in unemployment in in the sector $\left(d \log L_{i}<0\right)$ but no endogenous firm failures $\left(d \log M_{i}=d \log \bar{M}_{i}\right)$. On the other hand, sector $-i$ maintains full employment and experiences no failures.

To verify that this configuration is indeed an equilibrium, we compute

$$
d \log \lambda_{i}=\frac{(\theta-1)\left(1-\lambda_{i}\right)}{1-(\theta-1) \lambda_{i}} \frac{1}{\sigma-1} d \log \bar{M}_{i} .
$$

We must verify that

$$
0>d \log \lambda_{i}>d \log \bar{M}_{i} .
$$

The first inequality is verified as long as $\theta>1$ is not too large. The second inequality is verified if $\sigma>1$ is large enough and $\theta>1$ is not too large.

If these conditions are violated, then we can get a jump in the equilibrium outcome. Intuitively, in those cases, the shutdown triggers substitution away from $i$, and that substitution is so dramatic than it causes more firms to shutdown, and the process feeds on 
itself ad infinitum. Any level of $d \log L_{i}<0$ and $d \log M_{i}<d \log \bar{M}_{i}$ can then be supported as equilibria. Although we do not focus on it, this possibility illustrates how allowing for firm failures with increasing returns to scale can dramatically alter the model's behavior. Assuming the regularity conditions above are satisfied, the response of output is given by

$$
d \log Y=\lambda_{i} \frac{1}{\sigma-1} d \log \bar{M}_{i}+\frac{\left(\theta_{0}-1\right)\left(1-\lambda_{i}\right)}{1-\left(\theta_{0}-1\right) \lambda_{i}} \lambda_{i} \frac{1}{\sigma-1} d \log \bar{M}_{i}
$$

where the first term on the right-hand side is the direct effect of the shutdown and the second term is the amplification from the indirect effect of the shutdown which results in Keynesian unemployment in $i$. 